\title{
RESPIRATORY SYSTEM AFFECTION IN ACUTE POISONED CASES ADMITTED TO MENOUFIA POISON AND DEPENDENCE CONTROL CENTER (MPDCC), MENOUfIA UNIVERSITY hOSPITAL " A PROSPECTIVE STUDY "
}

\author{
Samy Abd El-Hady Hammad ${ }^{1}$, Azza Wagih Zanaty ${ }^{2}$, Ola Abdelhady Sweilum ${ }^{3}$, Fatma \\ Shaban Kandeel ${ }^{4}$, Reham Sobhi Abo-ayad ${ }^{5}$ \\ ${ }^{1-2}$ Professor of Forensic Medicine \& Clinical Toxicology, Menoufia University, ${ }^{3}$ Assistant \\ professor of Forensic Medicine \& Clinical Toxicology, Menoufia University, ${ }^{4}$ Lecturer of \\ Forensic Medicine \& Clinical Toxicology, Menoufia University, ${ }^{5}$ Demonstrator at Forensic \\ Medicine \& Clinical Toxicology, Menoufia University, Shebin Ei Kom, Egypt,
}

\begin{abstract}
Background: Acute poisoning is a common cause of admission to emergency departments (ED) and often needs treatment in the intensive care unit (ICU). Breathing and pulmonary complications are frequent causes of morbidity and mortality in acute poisoning. Aim of the work: To study respiratory system affection in acute poisoned cases as regarding: socio demographic pattern, their clinical manifestations, investigations and outcome according to poisoning severity score (PSS). Patients and methods: It is a prospective study that was conducted at Menoufia poison and dependence control center (MPDCC) through one year from the 1st October 2016 to the end of September 2017. All acute poisoned cases with respiratory system affection admitted to MPDCC were studied. The socio-demographic and clinical data were collected from each patient in a designed clinical toxicological sheet. The studied cases were classified according to the poisoning severity score. Results: the study included 116 patients. The incidence of respiratory system affection among total numbers of acute poisoned cases during the period of the study was $4.2 \%$. The most common age of cases was $<5$ years $(42.2 \%)$. Males outnumbered females $(54.3 \%$ versus $45.7 \%$ respectively). Regarding (PSS); $41.4 \%$ of cases were of moderate grade. As the causing substance of acute poisoning, cholinesterase inhibitors and corrosives were the most prominent causative agents $(30.2 \%$ and $27.6 \%$ respectively). Oral ingestion was the common route of administration (74.1\%). ICU admission was indicated for $29.3 \%$ of cases. Mortality rate constituted $10.3 \%$ of cases. Conclusion and recommendation: Respiratory system affection is an important cause of morbidity and mortality in acute poisoned cases. Respiratory system affection in acute poisoned cases can occur with a lot of poisons mostly with cholinesterase inhibitors and corrosives. Children less than 5 years were commonly affected. Therefore it is recommended to conduct educational programs in rural areas, restrict the availability
\end{abstract}


of pesticides and keeping them with other household products away from easy reach by children.

Keywords: acute poisoning, respiratory affection, cholinesterase inhibitors, corrosives.

\section{INTRODUCTION}

Morbidity and mortality due to acute poisoning is a major public health problem in many countries (Malangu and Ogunbanjo, 2009).

Respiratory complications and breathing difficulties are important risk factors in increasing morbidity and mortality rates in acute poisoned patients (Olson, 2012).

Respiratory system is commonly affected in acute poisoning as multiple poisons cause respiratory toxicity either due to poison itself by central or peripheral mechanisms or due to its complications of poisoning as aspiration pneumonia and prolonged ventilation (Stolbach and Hoffman , 2011).

Common poisons that cause respiratory system affection in acute state are anticholinesterases, organophossphorous nerve agents, drug overdose as opioids and other central depressants, animal bite particularly snake bite, carbon monoxide poisoning, hydrocarbons and corrosives (Little, 2015).

Acute poisoning associated with respiratory impairment is very dangerous and may needs treatment in intensive care unit (ICU) because they may cause life-threatening complications that may result in death (Jayakrishnan et al., 2012; Khodabandeh and Agin, 2016).

\section{AIM OF THE WORK}

This work aims to study respiratory system affection in acute poisoned cases as regarding: sociodemographic pattern, their clinical investigation, manifestations, according to poison severity score, treatment and outcome.

\section{PATIENTS \& METHODS}

This is a prospective study conducted on all acute poisoned patients suffered from respiratory system affection admitted to Menoufia Poison and Dependence Control Center (MPDCC) over one year during the period from the $1^{\text {st }}$ of October 2016 to the end of September 2017 after obtaining the ethical approval from the ethical committee of Faculty of Medicine, Menoufia University. Also, the permission of authority of Menoufia Poison and Dependence control center (MPDCC) which follows Menoufia University hospitals was taken before the study. The study included patients of both sexes and different age groups after taking their written valid consent from them or their guardians. Smokers and patients who had past history of chest disease were excluded from this study. Detailed history was taken regarding sociodemographic data (age, sex, residence, marital status and occupation), types of poison, clinical manifestations, investigations, classification of 
cases according to poison severity score (PSS), treatment and outcome. The studied cases were classified according to the poison severity score which is a four scale grading as (0) none, (1) minor, (2) moderate, (3) severe and (4) fatal (Person et al., 1998).

Laboratory (as arterial blood gases) and radiological (as chest $\mathrm{x}$ ray and computed tomography (CT) of chest) investigations were done when needed. Analytical toxicological tests (as thin layer chromatography, serum Pseudocholinesterase levels, and immunoassay rapid detection kits) were done when needed.
Stroboscopy was done when needed.

Statistical analysis:

Data were collected, tabulated and statistically analyzed using a personal computer with Statistical Package of Social Science (SPSS) version 20 and the following statistics were applied: descriptive statistics as percentage (\%) and analytic statistics as Chisquare test and fisher exact test. $\mathrm{P}$ value $>0.05$ was considered statistically non-significant. $\mathrm{P}$ value $<0.05$ was considered statistically significant. $\mathrm{P}$ value less than 0.001 was considered statistically highly significant (Hansen et al., 2009)

\section{$\underline{\text { RESULTS }}$}

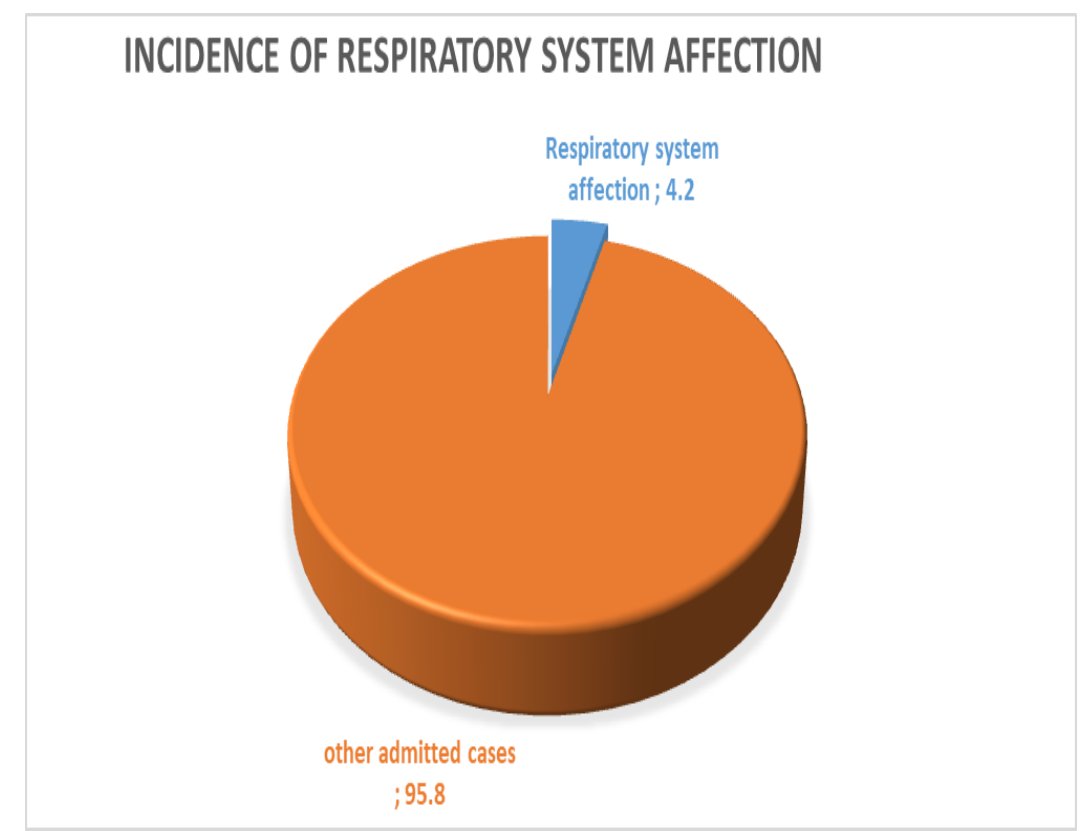

Figure (1): The incidence of respiratory system affection in acute poisoned cases during the period of the study was $4.2 \%$. 
Table (1): Distribution of respiratory system affection in the studied cases as regard sociodemographic data $(\mathrm{NO}=116)$ :

\begin{tabular}{|c|c|c|}
\hline \multirow[t]{2}{*}{\begin{tabular}{|l|} 
Sociodemographic data \\
\end{tabular}} & \multicolumn{2}{|c|}{$\begin{array}{l}\text { Studied group } \\
\text { NO=116 }\end{array}$} \\
\hline & NO & $\%$ \\
\hline Age in years & & \\
\hline$<5$ & 49 & 42.2 \\
\hline $5-<10$ & 1 & 0.9 \\
\hline $10-<20$ & 25 & 21.6 \\
\hline $20-40$ & 24 & 20.7 \\
\hline$>40$ & 17 & 14.7 \\
\hline Sex & & \\
\hline Male & 63 & 54.3 \\
\hline Female & 53 & 45.7 \\
\hline Residence & & \\
\hline Rural & 70 & 60.3 \\
\hline Urban & 46 & 39.7 \\
\hline Marital status & & \\
\hline child & 50 & 43.1 \\
\hline Single & 28 & 24.1 \\
\hline Married & 34 & 29.3 \\
\hline Widow & 3 & 2.6 \\
\hline Divorce & 1 & 0.9 \\
\hline Occupation & & \\
\hline Child & 50 & 43.1 \\
\hline Student & 22 & 19 \\
\hline Employer & 5 & 4.3 \\
\hline Worker & 22 & 19 \\
\hline Not working & 17 & 14.7 \\
\hline
\end{tabular}

Table (2): Statistical analysis Fisher's exact test of gender differentiation in age groups of the studied cases $(\mathrm{NO}=116)$ :

\begin{tabular}{|c|c|c|c|c|c|c|}
\hline \multirow[t]{2}{*}{ Age in years } & \multicolumn{2}{|c|}{$\begin{array}{l}\text { Male } \\
\text { NO =63 }\end{array}$} & \multicolumn{2}{|c|}{$\begin{array}{l}\text { Female } \\
\text { NO }=53\end{array}$} & \multirow[t]{2}{*}{$\begin{array}{l}\text { Fisher's exact } \\
\text { test }\end{array}$} & \multirow[t]{2}{*}{$\begin{array}{l}P \\
\text { value }\end{array}$} \\
\hline & NO & $\%$ & NO & $\%$ & & \\
\hline $\begin{array}{l}<5 \\
5-<10 \\
10-<20 \\
20-40 \\
>40\end{array}$ & $\begin{array}{l}24 \\
0 \\
7 \\
19 \\
13\end{array}$ & $\begin{array}{l}38.1 \\
0 \\
11.1 \\
30.2 \\
20.6\end{array}$ & $\begin{array}{l}25 \\
1 \\
18 \\
5 \\
4\end{array}$ & $\begin{array}{l}47.2 \\
1.9 \\
34 \\
9.4 \\
7.5\end{array}$ & 18.01 & $\begin{array}{c}0.001 \\
\text { HS }\end{array}$ \\
\hline
\end{tabular}

*P value $<0.05=$ significant $(\mathrm{S}) \quad * \mathrm{P}$ value $<0.001=$ highly significant $(\mathrm{HS})$

$* \mathrm{P}$ value $>0.05=$ non-significant $(\mathrm{NS}) \quad * \mathrm{NO}=$ number of cases 
Table (3): Percentage distribution of the studied cases according to poison history $(\mathrm{NO}=116)$ :

\begin{tabular}{|c|c|c|}
\hline 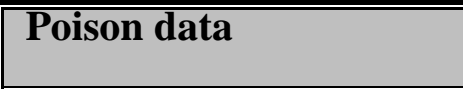 & $\begin{array}{r}\text { Stuc } \\
\text { Ne }\end{array}$ & \\
\hline & NO & $\%$ \\
\hline Poison type & & \\
\hline Cholinesterase inhibitors & 35 & 30.2 \\
\hline Drug overdose & 14 & 12.1 \\
\hline Co,gas inhalation & 11 & 9.5 \\
\hline Corrosive & 32 & 27.6 \\
\hline hydrocarbons & 13 & 11.2 \\
\hline Animal bite & 11 & \\
\hline Route of exposure & & \\
\hline Ingestion & 86 & 74.1 \\
\hline Inhalation & 15 & 12.9 \\
\hline Biting & 11 & 9.5 \\
\hline Injection & 1 & 0.9 \\
\hline Skin contamination & 3 & 2.6 \\
\hline Mode of exposure & & \\
\hline Accidental & 92 & 79.3 \\
\hline Suicide & 24 & 20.7 \\
\hline Time pass since exposure & & \\
\hline$<3$ hours & 83 & 71.6 \\
\hline 3-6 hours & 21 & 18.1 \\
\hline$>6$ hours & 12 & 10.3 \\
\hline Referral & & \\
\hline referred & 86 & 74.1 \\
\hline Not referred & 30 & 25.9 \\
\hline Place of exposure & & \\
\hline $\begin{array}{l}\text { Indoor } \\
\text { Outdoor }\end{array}$ & $\begin{array}{l}85 \\
31 \\
\end{array}$ & $\begin{array}{l}73.3 \\
26.7 \\
\end{array}$ \\
\hline Season of exposure & & \\
\hline Summer & 21 & 18.1 \\
\hline $\begin{array}{l}\text { Autumn } \\
\text { Winter }\end{array}$ & 23 & $\begin{array}{l}19.8 \\
302\end{array}$ \\
\hline $\begin{array}{l}\text { Winter } \\
\text { Spring }\end{array}$ & $\begin{array}{l}35 \\
37\end{array}$ & 31.9 \\
\hline
\end{tabular}

$* \mathrm{NO}=$ number of cases

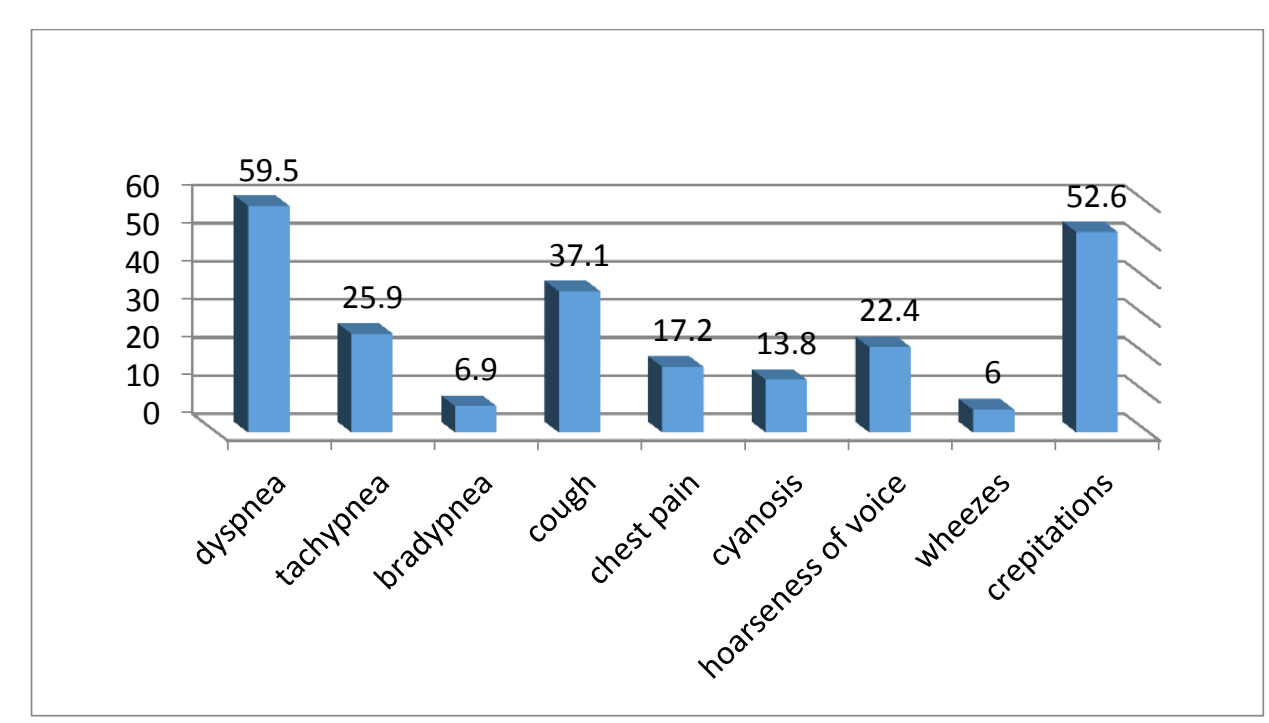

Figure (2): Distribution of clinical respiratory manifestations of studied cases at time of admission $(\mathrm{NO}=116)$ : 
Table (4): Statistical Analysis Fisher's Exact Tests of the Relation between Clinical Manifestation of studied cases and age $(\mathrm{NO}=116)$ :

\begin{tabular}{|c|c|c|c|c|c|c|c|c|c|c|c|c|}
\hline \multirow{3}{*}{$\begin{array}{l}\text { Clinical } \\
\text { manifestations }\end{array}$} & \multicolumn{10}{|c|}{ Age $(\mathrm{NO}=116)$} & \multirow{3}{*}{$\begin{array}{c}\text { Fisher's } \\
\text { exact } \\
\text { test }\end{array}$} & \multirow{3}{*}{$\begin{array}{l}P \\
\text { value }\end{array}$} \\
\hline & \multicolumn{2}{|c|}{$\begin{array}{c}<5 \\
\mathrm{NO}=49\end{array}$} & \multicolumn{2}{|c|}{$\begin{array}{l}5-<10 \\
\quad N O=1\end{array}$} & \multicolumn{2}{|c|}{$\begin{array}{l}10-<20 \\
N O=25\end{array}$} & \multicolumn{2}{|c|}{$\begin{array}{c}20-40 \\
\text { NO }=24\end{array}$} & \multicolumn{2}{|c|}{$\begin{array}{c}40> \\
N O=17\end{array}$} & & \\
\hline & NO & $\%$ & NO & $\%$ & NO & $\%$ & NO & $\%$ & NO & $\%$ & & \\
\hline $\begin{array}{l}\text { Tachypnea } \\
\text { Yes } \\
\text { No }\end{array}$ & $\begin{array}{l}14 \\
35\end{array}$ & $\begin{array}{l}28.6 \\
71.4\end{array}$ & $\begin{array}{l}0 \\
1\end{array}$ & $\begin{array}{c}0.0 \\
100.0\end{array}$ & $\begin{array}{c}4 \\
21\end{array}$ & $\begin{array}{l}16.0 \\
84.0\end{array}$ & $\begin{array}{c}6 \\
18\end{array}$ & $\begin{array}{l}25.0 \\
75.0\end{array}$ & $\begin{array}{c}6 \\
11\end{array}$ & $\begin{array}{l}35.3 \\
64.7\end{array}$ & 2.7 & $\begin{array}{l}0.65 \\
\text { NS }\end{array}$ \\
\hline $\begin{array}{l}\text { Dyspnea } \\
\text { Yes } \\
\text { No }\end{array}$ & $\begin{array}{l}21 \\
28\end{array}$ & $\begin{array}{l}42.9 \\
57.1\end{array}$ & $\begin{array}{l}1 \\
0\end{array}$ & $\begin{array}{r}100.0 \\
0.0\end{array}$ & $\begin{array}{c}20 \\
5\end{array}$ & $\begin{array}{l}80.0 \\
20.0\end{array}$ & $\begin{array}{c}15 \\
9\end{array}$ & $\begin{array}{l}62.5 \\
37.5\end{array}$ & $\begin{array}{c}12 \\
5\end{array}$ & $\begin{array}{l}70.6 \\
29.4\end{array}$ & 11.4 & $\begin{array}{c}0.01 \\
S\end{array}$ \\
\hline $\begin{array}{l}\text { Bradypnea } \\
\text { Yes } \\
\text { No }\end{array}$ & $\begin{array}{c}2 \\
47\end{array}$ & $\begin{array}{c}4.1 \\
95.9\end{array}$ & $\begin{array}{l}0 \\
1\end{array}$ & $\begin{array}{c}0.0 \\
100.0\end{array}$ & $\begin{array}{c}1 \\
24\end{array}$ & $\begin{array}{c}4.0 \\
96.0\end{array}$ & $\begin{array}{c}3 \\
21\end{array}$ & $\begin{array}{l}12.5 \\
87.5\end{array}$ & $\begin{array}{c}2 \\
15\end{array}$ & $\begin{array}{l}11.8 \\
88.2\end{array}$ & 3.9 & $\begin{array}{c}0.42 \\
\mathrm{NS}\end{array}$ \\
\hline $\begin{array}{l}\text { Cough } \\
\text { Yes } \\
\text { No }\end{array}$ & $\begin{array}{l}28 \\
21\end{array}$ & $\begin{array}{l}57.1 \\
42.9\end{array}$ & $\begin{array}{l}1 \\
0\end{array}$ & $\begin{array}{r}100.0 \\
0.0\end{array}$ & $\begin{array}{c}2 \\
23\end{array}$ & $\begin{array}{c}8.0 \\
92.0\end{array}$ & $\begin{array}{c}5 \\
19\end{array}$ & $\begin{array}{l}20.8 \\
79.2\end{array}$ & $\begin{array}{c}7 \\
10\end{array}$ & $\begin{array}{l}41.2 \\
58.8\end{array}$ & 22.8 & $\begin{array}{c}<0.001 \\
\mathrm{HS}\end{array}$ \\
\hline $\begin{array}{l}\text { Chest pain } \\
\text { Yes } \\
\text { No }\end{array}$ & $\begin{array}{c}2 \\
47 \\
\end{array}$ & $\begin{array}{c}4.1 \\
95.9 \\
\end{array}$ & $\begin{array}{l}1 \\
0 \\
\end{array}$ & $\begin{array}{r}100.0 \\
0.0 \\
\end{array}$ & $\begin{array}{c}5 \\
20 \\
\end{array}$ & $\begin{array}{l}20.0 \\
80.0 \\
\end{array}$ & $\begin{array}{c}8 \\
16 \\
\end{array}$ & $\begin{array}{l}33.3 \\
66.7 \\
\end{array}$ & $\begin{array}{c}4 \\
13 \\
\end{array}$ & $\begin{array}{l}23.5 \\
76.5 \\
\end{array}$ & 15.6 & $\begin{array}{c}0.001 \\
\mathrm{~S} \\
\end{array}$ \\
\hline $\begin{array}{l}\text { Cyanosis } \\
\text { Yes } \\
\text { No }\end{array}$ & $\begin{array}{c}3 \\
46\end{array}$ & $\begin{array}{c}6.1 \\
93.9\end{array}$ & $\begin{array}{l}0 \\
1\end{array}$ & $\begin{array}{c}0.0 \\
100.0\end{array}$ & $\begin{array}{l}3 \\
22\end{array}$ & $\begin{array}{l}12.0 \\
88.0\end{array}$ & $\begin{array}{c}5 \\
19\end{array}$ & $\begin{array}{l}20.8 \\
79.2\end{array}$ & $\begin{array}{c}5 \\
12\end{array}$ & $\begin{array}{l}29.4 \\
70.6\end{array}$ & 7.5 & $\begin{array}{c}0.09 \\
\text { NS }\end{array}$ \\
\hline $\begin{array}{l}\text { Dyspnea \& } \\
\text { hoarseness of } \\
\text { voice } \\
\text { Yes } \\
\text { No } \\
\end{array}$ & $\begin{array}{c}9 \\
40\end{array}$ & $\begin{array}{l}18.4 \\
81.6\end{array}$ & $\begin{array}{l}0 \\
1\end{array}$ & $\begin{array}{c}0.0 \\
100.0\end{array}$ & $\begin{array}{c}4 \\
21\end{array}$ & $\begin{array}{l}16.0 \\
84.0\end{array}$ & $\begin{array}{l}12 \\
12\end{array}$ & $\begin{array}{l}50.0 \\
50.0\end{array}$ & $\begin{array}{c}1 \\
16\end{array}$ & $\begin{array}{c}5.9 \\
94.1\end{array}$ & 12.8 & $\begin{array}{l}0.008 \\
S\end{array}$ \\
\hline $\begin{array}{l}\text { Chest } \\
\text { examination } \\
\text { Normal } \\
\text { Wheezes } \\
\text { crepitations }\end{array}$ & $\begin{array}{c}16 \\
5 \\
28 \\
\end{array}$ & $\begin{array}{l}32.7 \\
10.2 \\
57.1 \\
\end{array}$ & $\begin{array}{l}0 \\
0 \\
1 \\
\end{array}$ & $\begin{array}{c}0.0 \\
0.0 \\
100.0 \\
\end{array}$ & $\begin{array}{c}6 \\
0 \\
19 \\
\end{array}$ & $\begin{array}{c}24.0 \\
0.0 \\
76.0 \\
\end{array}$ & $\begin{array}{c}16 \\
1 \\
7 \\
\end{array}$ & $\begin{array}{c}66.7 \\
4.2 \\
29.2 \\
\end{array}$ & $\begin{array}{c}10 \\
1 \\
6 \\
\end{array}$ & $\begin{array}{c}58.8 \\
5.9 \\
35.3 \\
\end{array}$ & 18.02 & $\begin{array}{c}0.01 \\
\mathrm{~S}\end{array}$ \\
\hline
\end{tabular}

*NS=non- significant $*$ S=significant $* \mathrm{HS}=$ high significant $* \mathrm{NO}=$ number of cases 
Table (5): Statistical analysis fisher's exact test of the relation between the clinical presentations of the studied cases and the causative poisons $(\mathrm{NO}=116)$ :

\begin{tabular}{|c|c|c|c|c|c|c|c|c|c|c|c|c|c|c|}
\hline \multirow{3}{*}{$\begin{array}{l}\text { Clinical } \\
\text { manifestation } \\
\text { S }\end{array}$} & \multicolumn{10}{|c|}{ Poison type $(\mathrm{NO}=116)$} & & & \multirow{3}{*}{$\begin{array}{l}\text { Fishe } \\
\text { r's } \\
\text { exact } \\
\text { test }\end{array}$} & \multirow{3}{*}{$\begin{array}{l}P \\
\text { value }\end{array}$} \\
\hline & \multicolumn{2}{|c|}{$\begin{array}{l}\text { Anti- } \\
\text { cholinester } \\
\text { ase } \\
\quad \text { NO }=35\end{array}$} & \multicolumn{2}{|c|}{$\begin{array}{c}\text { Drug } \\
\text { overdose } \\
\mathrm{NO}=14\end{array}$} & \multicolumn{2}{|c|}{$\begin{array}{c}\text { CO, gas } \\
\text { inhalation } \\
\text { NO=11 }\end{array}$} & \multicolumn{2}{|c|}{$\begin{array}{c}\text { corrosives } \\
\text { NO }=32\end{array}$} & \multicolumn{2}{|c|}{$\begin{array}{l}\text { volatiles } \\
\mathrm{NO}=13\end{array}$} & \multicolumn{2}{|c|}{$\begin{array}{c}\text { Animal } \\
\text { Bite } \\
\text { NO=11 }\end{array}$} & & \\
\hline & NO & $\%$ & NO & $\%$ & NO & $\%$ & $\begin{array}{l}\mathbf{N} \\
\mathbf{O}\end{array}$ & $\%$ & NO & $\%$ & $\begin{array}{l}\mathbf{N} \\
\mathbf{O}\end{array}$ & $\%$ & & \\
\hline $\begin{array}{l}\text { dyspnea } \\
\text { Yes } \\
\text { No }\end{array}$ & $\begin{array}{c}26 \\
9\end{array}$ & $\begin{array}{l}74.3 \\
25.7\end{array}$ & $\begin{array}{c}3 \\
11\end{array}$ & $\begin{array}{l}21.4 \\
78.6\end{array}$ & $\begin{array}{l}5 \\
6\end{array}$ & $\begin{array}{l}45.5 \\
54.5\end{array}$ & $\begin{array}{l}17 \\
15\end{array}$ & $\begin{array}{l}53.1 \\
46.9\end{array}$ & $\begin{array}{l}6 \\
7\end{array}$ & $\begin{array}{l}46.2 \\
53.8\end{array}$ & $\begin{array}{c}11 \\
0\end{array}$ & $\begin{array}{c}100.0 \\
0.0\end{array}$ & 21.7 & $\begin{array}{c}<0.00 \\
1 \\
\text { HS }\end{array}$ \\
\hline $\begin{array}{l}\text { Tachypnea } \\
\text { Yes } \\
\text { No }\end{array}$ & $\begin{array}{c}4 \\
31\end{array}$ & $\begin{array}{l}11.4 \\
88.6\end{array}$ & $\begin{array}{c}0 \\
14\end{array}$ & $\begin{array}{l}0.0 \\
0.0\end{array}$ & $\begin{array}{l}7 \\
4\end{array}$ & $\begin{array}{l}63.6 \\
36.4\end{array}$ & $\begin{array}{c}7 \\
25\end{array}$ & $\begin{array}{l}21.9 \\
78.1\end{array}$ & $\begin{array}{l}8 \\
5\end{array}$ & $\begin{array}{l}61.5 \\
38.5\end{array}$ & $\begin{array}{l}4 \\
7\end{array}$ & $\begin{array}{l}36.4 \\
63.6\end{array}$ & 25.2 & $\begin{array}{c}<0.00 \\
1 \\
\text { HS } \\
\end{array}$ \\
\hline $\begin{array}{l}\text { bradypnea } \\
\text { Yes } \\
\text { No }\end{array}$ & $\begin{array}{c}0 \\
35\end{array}$ & $\begin{array}{c}0.0 \\
100 . \\
0 \\
\end{array}$ & $\begin{array}{c}4 \\
10\end{array}$ & $\begin{array}{l}28.6 \\
71.4\end{array}$ & $\begin{array}{c}0 \\
11\end{array}$ & $\begin{array}{c}0.0 \\
100.0\end{array}$ & $\begin{array}{c}0 \\
32\end{array}$ & $\begin{array}{l}0.0 \\
100\end{array}$ & $\begin{array}{c}0 \\
13\end{array}$ & $\begin{array}{r}0.0 \\
100.0\end{array}$ & $\begin{array}{l}4 \\
7\end{array}$ & $\begin{array}{l}36.4 \\
63.6\end{array}$ & 21.2 & $\begin{array}{c}<0.00 \\
1 \\
\text { HS } \\
\end{array}$ \\
\hline $\begin{array}{l}\text { Cough } \\
\text { Yes } \\
\text { No }\end{array}$ & $\begin{array}{c}3 \\
32\end{array}$ & $\begin{array}{c}8.6 \\
91.4\end{array}$ & $\begin{array}{c}0 \\
14\end{array}$ & $\begin{array}{r}0.0 \\
100.0\end{array}$ & $\begin{array}{l}3 \\
8\end{array}$ & $\begin{array}{l}27.3 \\
72.7\end{array}$ & $\begin{array}{c}25 \\
7\end{array}$ & $\begin{array}{l}78.1 \\
21.9\end{array}$ & $\begin{array}{c}11 \\
2\end{array}$ & $\begin{array}{l}84.6 \\
15.4\end{array}$ & $\begin{array}{c}1 \\
10\end{array}$ & $\begin{array}{c}9.1 \\
90.9\end{array}$ & 62.3 & $\begin{array}{c}<0.00 \\
1 \\
\text { HS } \\
\end{array}$ \\
\hline $\begin{array}{l}\text { Chest pain } \\
\text { Yes } \\
\text { No }\end{array}$ & $\begin{array}{c}3 \\
32\end{array}$ & $\begin{array}{c}8.6 \\
91.4\end{array}$ & $\begin{array}{c}1 \\
13\end{array}$ & $\begin{array}{r}7.1 \\
92.9\end{array}$ & $\begin{array}{l}2 \\
9\end{array}$ & $\begin{array}{l}18.2 \\
81.8\end{array}$ & $\begin{array}{l}11 \\
21\end{array}$ & $\begin{array}{l}34.4 \\
65.6\end{array}$ & $\begin{array}{c}0 \\
13\end{array}$ & $\begin{array}{c}0.0 \\
100.0\end{array}$ & $\begin{array}{l}3 \\
8\end{array}$ & $\begin{array}{l}27.3 \\
72.7\end{array}$ & 11.8 & $\begin{array}{c}0.01 \\
\mathrm{~S}\end{array}$ \\
\hline $\begin{array}{l}\text { Cyanosis } \\
\text { Yes } \\
\text { No } \\
\end{array}$ & $\begin{array}{c}6 \\
29\end{array}$ & $\begin{array}{l}17.1 \\
82.9\end{array}$ & $\begin{array}{c}4 \\
10\end{array}$ & $\begin{array}{l}28.6 \\
71.4\end{array}$ & $\begin{array}{l}0 \\
11\end{array}$ & $\begin{array}{c}0.0 \\
100.0\end{array}$ & $\begin{array}{c}0 \\
32\end{array}$ & $\begin{array}{r}0.0 \\
100\end{array}$ & $\begin{array}{c}1 \\
12\end{array}$ & $\begin{array}{c}7.7 \\
92.3\end{array}$ & $\begin{array}{l}5 \\
6\end{array}$ & $\begin{array}{l}45.5 \\
54.5\end{array}$ & 17.9 & $\begin{array}{c}0.001 \\
\mathrm{~S}\end{array}$ \\
\hline $\begin{array}{l}\text { Dyspnea \& } \\
\text { hoarseness of } \\
\text { voice } \\
\text { Yes } \\
\text { No }\end{array}$ & $\begin{array}{c}0 \\
35\end{array}$ & $\begin{array}{c}0.0 \\
100 . \\
0\end{array}$ & $\begin{array}{c}0 \\
14\end{array}$ & $\begin{array}{c}0.0 \\
100.0\end{array}$ & $\begin{array}{c}0 \\
11\end{array}$ & $\begin{array}{c}0.0 \\
100.0\end{array}$ & $\begin{array}{l}16 \\
16\end{array}$ & $\begin{array}{l}50.0 \\
50.0\end{array}$ & $\begin{array}{c}0 \\
13\end{array}$ & $\begin{array}{c}0.0 \\
100.0\end{array}$ & $\begin{array}{c}10 \\
1\end{array}$ & $\begin{array}{c}90.9 \\
9.1\end{array}$ & 60.7 & $\begin{array}{c}<0.00 \\
1 \\
\text { HS }\end{array}$ \\
\hline $\begin{array}{l}\text { Chest } \\
\text { examination } \\
\text { Normal } \\
\text { Wheezes } \\
\text { Crepitations }\end{array}$ & $\begin{array}{c}3 \\
0 \\
32\end{array}$ & $\begin{array}{c}8.6 \\
0.0 \\
91.4\end{array}$ & $\begin{array}{c}12 \\
0 \\
2\end{array}$ & $\begin{array}{c}85.7 \\
0.0 \\
14.3\end{array}$ & $\begin{array}{l}9 \\
0 \\
2\end{array}$ & $\begin{array}{c}81.8 \\
0.0 \\
18.2\end{array}$ & $\begin{array}{c}7 \\
4 \\
21\end{array}$ & $\begin{array}{l}21.9 \\
12.5 \\
65.6\end{array}$ & $\begin{array}{l}7 \\
3 \\
3\end{array}$ & $\begin{array}{l}53.8 \\
23.1 \\
23.1\end{array}$ & $\begin{array}{c}10 \\
0 \\
1\end{array}$ & $\begin{array}{c}90.9 \\
0.0 \\
9.1\end{array}$ & 63.1 & $\begin{array}{c}<0.00 \\
1 \\
\mathrm{HS}\end{array}$ \\
\hline
\end{tabular}




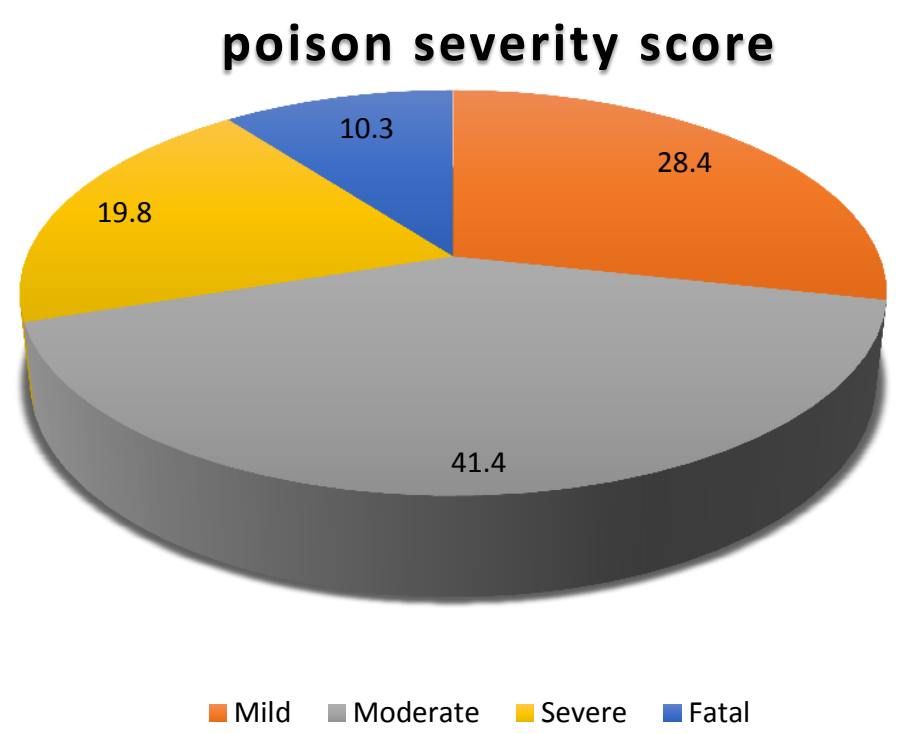

Figure (3): Percentage distribution of severity grades of the studied cases according to poison severity score (PSS) 
Table (6): Distribution of the investigations done to the studied cases.

\begin{tabular}{|c|c|c|}
\hline \multirow[t]{2}{*}{ Investigations } & \multicolumn{2}{|c|}{ Studied groups } \\
\hline & NO & $\%$ \\
\hline \multicolumn{3}{|l|}{ Arterial blood gases (done to $70.7 \%$ of cases) } \\
\hline Normal & 5 & 6.1 \\
\hline Acidosis (respiratory \& metabolic) & 33 & 40.2 \\
\hline Respiratory alkalosis & 16 & 19.5 \\
\hline Mixed metabolic acidosis \& respiratory alkalosis & 24 & 29.3 \\
\hline Hypoxia & 4 & 4.9 \\
\hline total & 82 & 100.0 \\
\hline \multicolumn{3}{|c|}{ Thin layer chromatography(TLC) (done to $32.8 \%$ of cases) } \\
\hline positive & 32 & 84.2 \\
\hline Negative & 6 & 18.8 \\
\hline Total & 38 & 100.0 \\
\hline \multicolumn{3}{|l|}{ Pseudocholinesterase level (done to $25.9 \%$ of cases) } \\
\hline Normal & 1 & 3.3 \\
\hline Decreased & 26 & 96.7 \\
\hline Total & 27 & 100.0 \\
\hline \multicolumn{3}{|c|}{ Rapid detection kit (immunoassay) (done to $13.8 \%$ of cases) } \\
\hline positive & 12 & 75.0 \\
\hline negative & 4 & 25.0 \\
\hline Total & 16 & 100.0 \\
\hline \multicolumn{3}{|l|}{ Chest X-ray (done to $67.2 \%$ of cases) } \\
\hline Normal & 36 & 46.2 \\
\hline Abnormal & 42 & 53.8 \\
\hline Total & 78 & 100.0 \\
\hline \multicolumn{3}{|l|}{ Chest CT (done to $15.5 \%$ of cases) } \\
\hline Normal & 2 & 11.1 \\
\hline Abnormal & 16 & 88.9 \\
\hline Total & 18 & 100.0 \\
\hline
\end{tabular}

No $=$ number of cases 


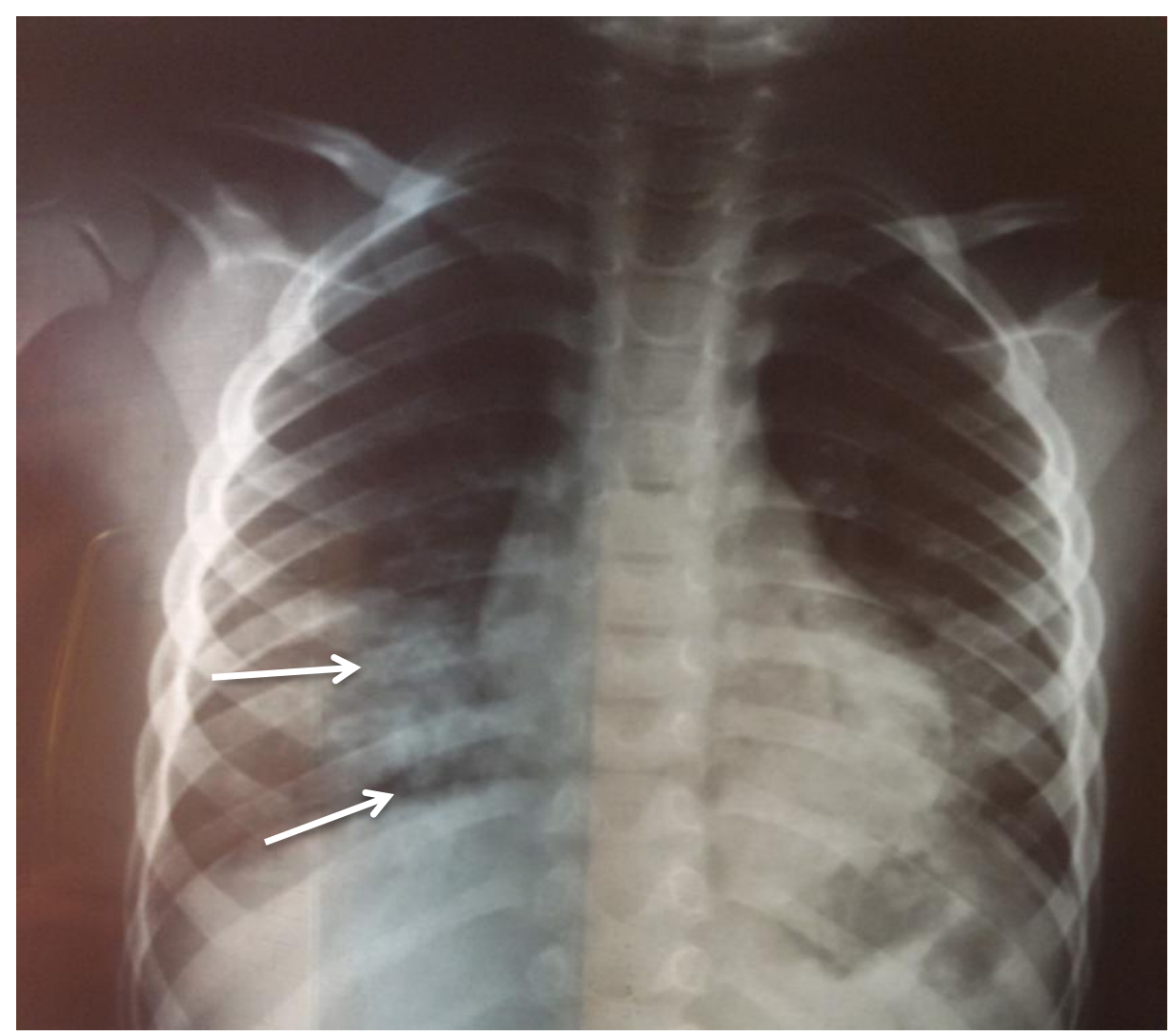

Figure (4): Chest x-ray of male patient 3 years old with kerosene toxicity showing right basal pneumonic patches (arrows)

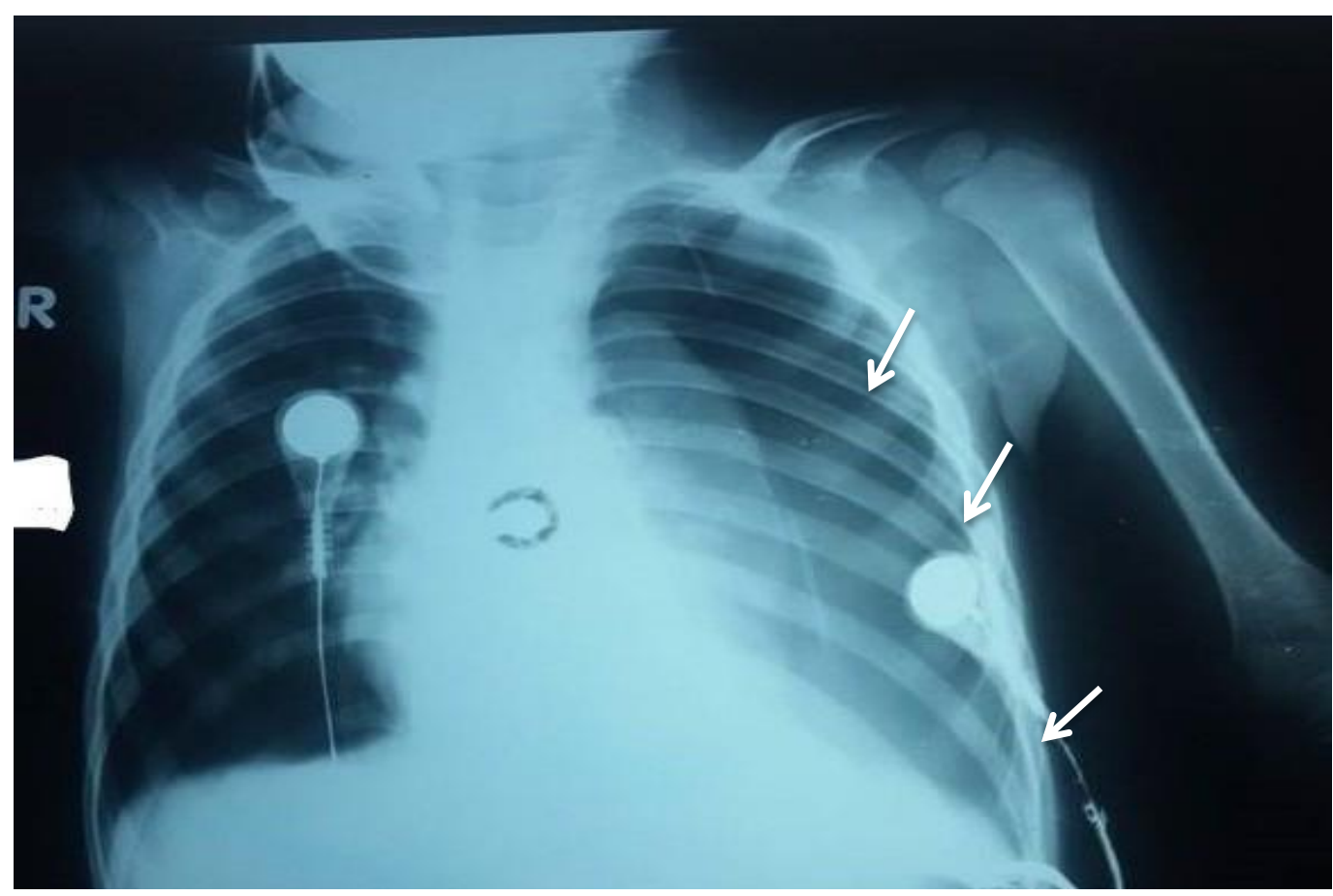

Figure (5): Chest $\mathrm{x}$-ray of female patient 3 years old with corrosive toxicity showing left sided pneumothorax (arrows). 


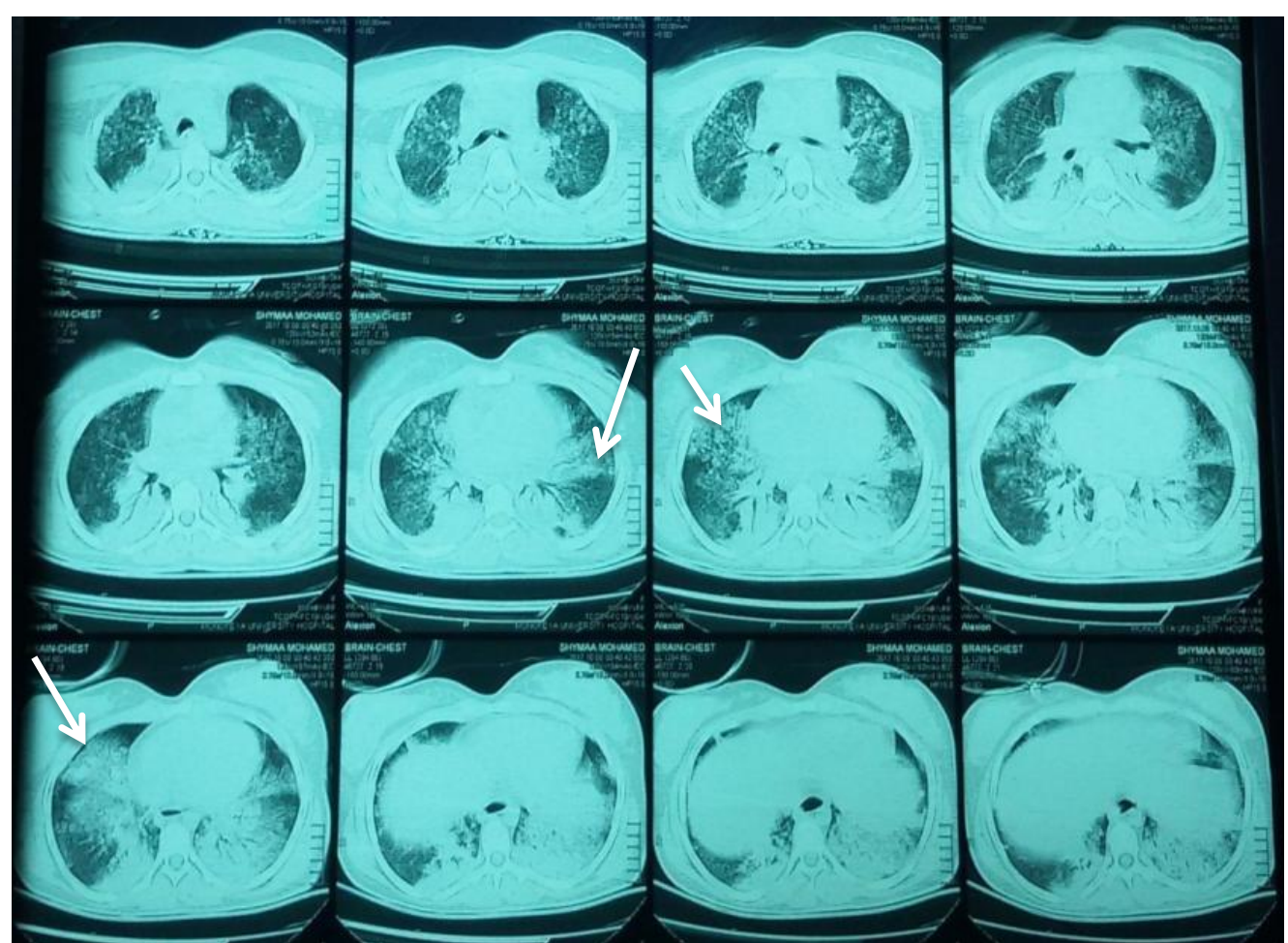

Figure (6): C.T. chest of female patient 18 years old with insecticide toxicity (white powder ingestion) showing bilateral pulmonary consolidation (arrows) 


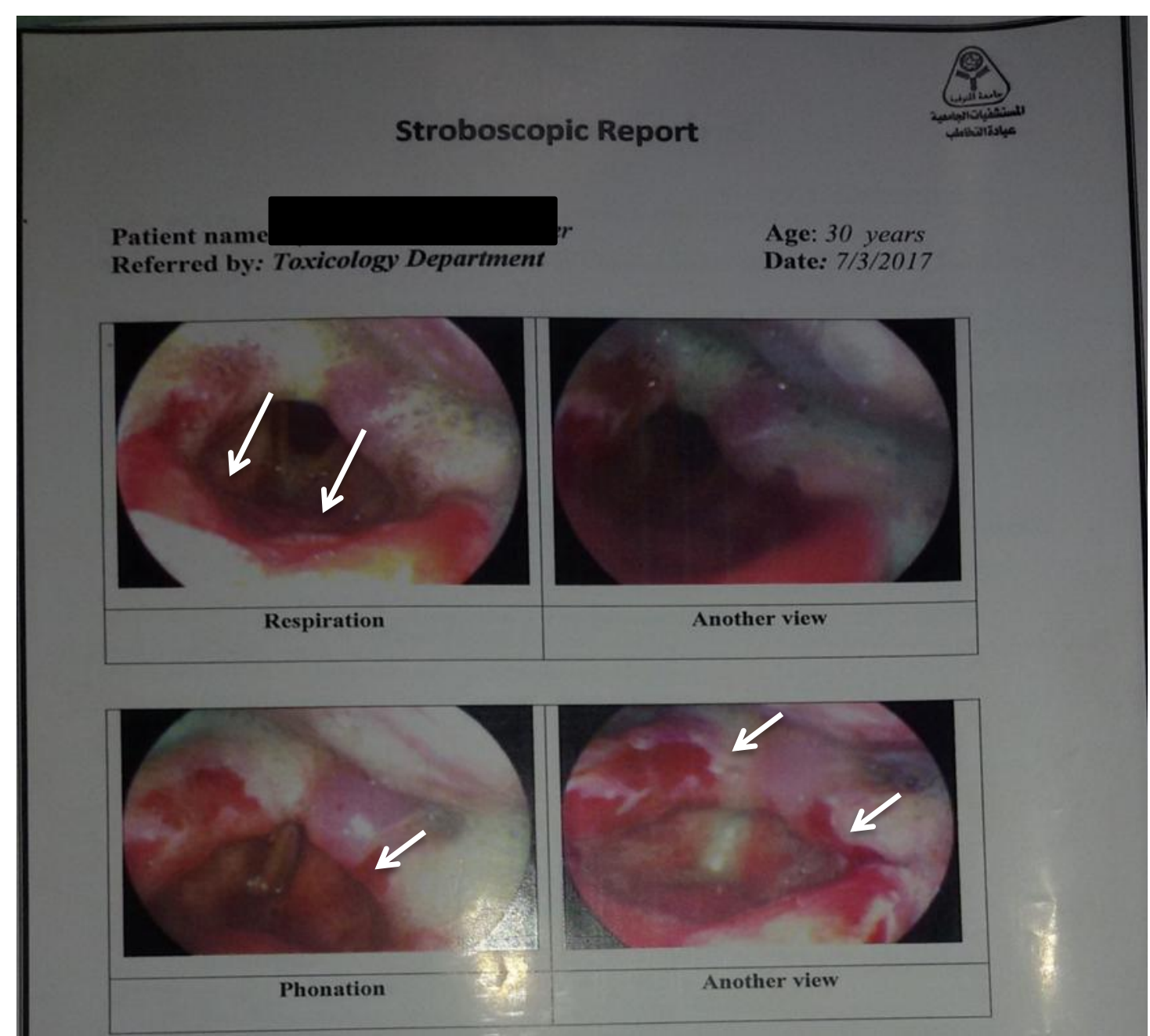

Figure (7): shows stroboscopic examination of male patient 30 years old showing highly edematous congested supraglottic area with macerated ulcerated mucosa and congested edematous subglottic area (laryngitis) after corrosive ingestion (arrows). 
Table (7): Statistical Analysis Chi-square $\left(X^{2}\right)$ and Fisher's Exact Tests of the Relation between radiological finding of the studied cases, type of poison and age $(\mathrm{NO}=116)$ :

\begin{tabular}{|c|c|c|c|c|c|c|c|c|c|c|c|c|}
\hline \multirow[t]{3}{*}{ Clinical manifestations } & \multicolumn{10}{|c|}{ Age $(\mathrm{NO}=116)$} & \multirow{3}{*}{$\begin{array}{c}\text { Fisher's } \\
\text { exact } \\
\text { test }\end{array}$} & \multirow[t]{3}{*}{ Pvalue } \\
\hline & \multicolumn{2}{|c|}{$\begin{array}{r}<5 \\
\text { NO }=49\end{array}$} & \multicolumn{2}{|c|}{$\begin{array}{l}5-<10 \\
\mathrm{NO}=1\end{array}$} & \multicolumn{2}{|c|}{$\begin{array}{l}10-<20 \\
\mathrm{NO}=25\end{array}$} & \multicolumn{2}{|c|}{$\begin{array}{c}20-40 \\
\text { NO }=24\end{array}$} & \multicolumn{2}{|c|}{$\begin{array}{c}>40 \\
\mathrm{NO}=17\end{array}$} & & \\
\hline & NO & $\%$ & NO & $\%$ & NO & $\%$ & NO & $\%$ & NO & $\%$ & & \\
\hline $\begin{array}{l}\text { Chest X-ray } \\
\text { Normal }\end{array}$ & 15 & 30.6 & 1 & 100 & 5 & 20 & 9 & 37.5 & 6 & 35.3 & & 0.31 \\
\hline Abnormal & 20 & 40.8 & 0 & 0 & 7 & 28 & 7 & 29.2 & 8 & 47.1 & 8.9 & NS \\
\hline Not done & 14 & 28.6 & 0 & 0 & 13 & 52 & 8 & 33.3 & 3 & 17.6 & & \\
\hline Chest CT & & & & & & & & & & & & \\
\hline Normal & 0 & 0 & 0 & 0 & 1 & 4 & 1 & 4.2 & 0 & 0 & & 0.02 \\
\hline Abnormal & 2 & 4.1 & 0 & 0 & 6 & 24 & 3 & 12.5 & 5 & 29.4 & 16.1 & $\mathrm{~S}$ \\
\hline Not done & 47 & 95.9 & 1 & 100 & 18 & 72 & 20 & 83.3 & 12 & 70.6 & & \\
\hline Poison type & & & & & & & & & & & & \\
\hline Anticholinesterase & 9 & 18.4 & 0 & 0 & 16 & 64 & 4 & 16.7 & 6 & 35.3 & & \\
\hline Drug overdose & 7 & 14.3 & 0 & 0 & 2 & 8 & 3 & 12.5 & 2 & 11.8 & & 0.03 \\
\hline Co , gas inhalation & 0 & 0 & 0 & 0 & 3 & 12 & 5 & 20.8 & 3 & 17.6 & 15.7 & $\mathrm{~S}$ \\
\hline Corrosive & 20 & 40.8 & 1 & 100 & 2 & 8 & 5 & 20.8 & 4 & 23.5 & & \\
\hline hydrocarbons & 13 & 26.5 & 0 & 0 & 0 & 0 & 0 & 0 & 0 & 0 & & \\
\hline Animal bite & 0 & 0 & 0 & 0 & 2 & 8 & 7 & 29.2 & 2 & 11.8 & & \\
\hline
\end{tabular}

*NS=non- significant $\quad * \mathrm{~S}=$ significant $\quad{ }^{*} \mathrm{HS}=$ high significant $* \mathrm{NO}=$ number of cases

Table (8): Statistical Analysis Fisher's Exact Test of the Relation between $\mathrm{ABG}$ and Age, sex, poison type $(\mathrm{NO}=116)$ :

\begin{tabular}{|c|c|c|c|c|c|c|c|c|c|c|c|c|c|c|}
\hline & \multicolumn{12}{|c|}{ ABG (NO =116) } & \multirow{3}{*}{$\begin{array}{c}\text { Fisher's } \\
\text { exact test }\end{array}$} & \multirow{3}{*}{$\begin{array}{r}P \\
\text { value }\end{array}$} \\
\hline & \multicolumn{2}{|c|}{$\begin{array}{c}\text { Normal } \\
\text { NO }=5\end{array}$} & \multicolumn{2}{|c|}{$\begin{array}{c}\text { Acidosis } \\
\text { NO }=33\end{array}$} & \multicolumn{2}{|c|}{$\begin{array}{c}\text { Resp. } \\
\text { alkalosis } \\
\text { NO=16 }\end{array}$} & \multicolumn{2}{|c|}{$\begin{array}{l}\text { Mix metabolic } \\
\text { acidosis } \\
\text { \&respiratory } \\
\text { alkalosis } \\
\text { NO=24 }\end{array}$} & \multicolumn{2}{|c|}{$\begin{array}{l}\text { Not done } \\
\text { No=34 }\end{array}$} & \multicolumn{2}{|c|}{$\begin{array}{r}\text { Hypoxia } \\
\text { No=4 }\end{array}$} & & \\
\hline & $\mathbf{N}$ & $\%$ & $\mathbf{N}$ & $\%$ & $\mathbf{N}$ & $\%$ & $\mathbf{N}$ & $\%$ & $\mathbf{N}$ & $\%$ & $\mathbf{N}$ & $\%$ & & \\
\hline $\begin{array}{l}\text { Sex } \\
\text { Male } \\
\text { Female }\end{array}$ & $\begin{array}{l}3 \\
2\end{array}$ & $\begin{array}{l}60 \\
40\end{array}$ & $\begin{array}{l}20 \\
13\end{array}$ & $\begin{array}{l}60 \\
40\end{array}$ & $\begin{array}{l}9 \\
7\end{array}$ & $\begin{array}{l}56.3 \\
43.7\end{array}$ & $\begin{array}{l}10 \\
14\end{array}$ & $\begin{array}{l}41.7 \\
58.3\end{array}$ & $\begin{array}{l}18 \\
16\end{array}$ & $\begin{array}{l}52.9 \\
47.1\end{array}$ & $\begin{array}{l}3 \\
1\end{array}$ & $\begin{array}{l}75 \\
25\end{array}$ & 2.9 & $\begin{array}{c}0.74 \\
\text { NS }\end{array}$ \\
\hline $\begin{array}{l}\text { Age in years } \\
<5 \\
5-<10 \\
10-<20 \\
20-40 \\
>40\end{array}$ & $\begin{array}{l}0 \\
0 \\
1 \\
1 \\
3\end{array}$ & $\begin{array}{c}0.0 \\
0.0 \\
20.0 \\
20.0 \\
60.0\end{array}$ & $\begin{array}{c}10 \\
1 \\
6 \\
10 \\
6\end{array}$ & $\begin{array}{c}30.3 \\
3.0 \\
18.2 \\
30.3 \\
18.2\end{array}$ & $\begin{array}{l}7 \\
0 \\
3 \\
4 \\
2\end{array}$ & $\begin{array}{c}43.8 \\
0.0 \\
18.8 \\
25.0 \\
12.5 \\
\end{array}$ & $\begin{array}{l}7 \\
0 \\
9 \\
3 \\
5\end{array}$ & $\begin{array}{c}29.2 \\
0.0 \\
37.5 \\
12 . .5 \\
20.8\end{array}$ & $\begin{array}{l}25 \\
0 \\
5 \\
4 \\
0\end{array}$ & $\begin{array}{c}73.5 \\
0.0 \\
14.7 \\
11.8 \\
0.0\end{array}$ & $\begin{array}{l}0 \\
0 \\
1 \\
2 \\
1\end{array}$ & $\begin{array}{c}0.0 \\
0.0 \\
25.0 \\
50.0 \\
250\end{array}$ & 22.5 & $\begin{array}{c}0.002 \\
\mathrm{~S}\end{array}$ \\
\hline $\begin{array}{l}\text { Poison type } \\
\text { Anticholinesterase } \\
\text { Drug overdose } \\
\text { Co ,gas inhalation } \\
\text { Corrosive } \\
\text { hydrocarbons } \\
\text { Animal bite }\end{array}$ & $\begin{array}{l}0 \\
0 \\
0 \\
4 \\
0 \\
1\end{array}$ & $\begin{array}{c}0 \\
0 \\
0 \\
80 \\
0 \\
20\end{array}$ & $\begin{array}{l}13 \\
12 \\
1 \\
2 \\
0 \\
5\end{array}$ & $\begin{array}{c}39.4 \\
36.4 \\
3 \\
6.1 \\
0 \\
15.2\end{array}$ & $\begin{array}{l}1 \\
0 \\
5 \\
4 \\
4 \\
2\end{array}$ & $\begin{array}{c}6.3 \\
0 \\
31.3 \\
25 \\
25 \\
12.5\end{array}$ & $\begin{array}{c}13 \\
1 \\
4 \\
3 \\
2 \\
1\end{array}$ & $\begin{array}{c}54.2 \\
4.2 \\
16.7 \\
12.5 \\
8.3 \\
4.2\end{array}$ & $\begin{array}{c}8 \\
0 \\
0 \\
19 \\
7 \\
0\end{array}$ & $\begin{array}{c}23.5 \\
0 \\
0 \\
55.9 \\
20.6 \\
0\end{array}$ & $\begin{array}{l}0 \\
1 \\
1 \\
0 \\
0 \\
2\end{array}$ & $\begin{array}{c}0 \\
25 \\
25 \\
0 \\
0 \\
50\end{array}$ & 29.2 & $\begin{array}{l}0.001 \\
S\end{array}$ \\
\hline
\end{tabular}

${ }^{*} \mathrm{NS}=$ non- significant $\quad{ }^{*} \mathrm{~S}=$ significant $\quad * \mathrm{HS}=$ high significant $* \mathrm{NO}=$ number of cases 


\section{outcome}

completely cured

n cured with complication

discharded against medical advice

death

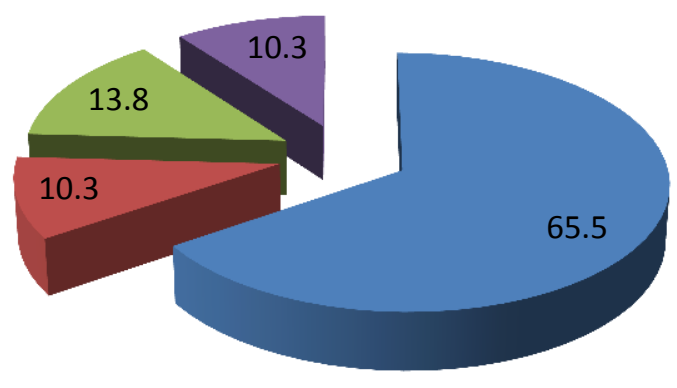

Figure (8): Percentage distribution of studied cases according to their outcome.

Table (9): Statistical Analysis Fisher's Exact Test of the Relation between rout of exposure poison severity score, Outcome and ICU admission ( $\mathrm{NO}=116)$ :

\begin{tabular}{|c|c|c|c|c|c|c|c|c|c|c|c|c|}
\hline & \multicolumn{10}{|c|}{ Rout of exposure $(\mathrm{NO}=116)$} & \multirow{3}{*}{$\begin{array}{c}\text { Fisher's } \\
\text { exact } \\
\text { test }\end{array}$} & \multirow{3}{*}{$\begin{array}{c}P \\
\text { value }\end{array}$} \\
\hline & \multicolumn{2}{|c|}{$\begin{array}{c}\text { Ingestion } \\
\mathrm{NO}=86\end{array}$} & \multicolumn{2}{|c|}{$\begin{array}{c}\text { Inhalation } \\
\mathrm{NO}=15\end{array}$} & \multicolumn{2}{|c|}{$\begin{array}{c}\text { biting } \\
\mathrm{NO}=11\end{array}$} & \multicolumn{2}{|c|}{$\begin{array}{c}\text { Injection } \\
\text { NO=1 }\end{array}$} & \multicolumn{2}{|c|}{$\begin{array}{l}\text { Skin } \\
\text { contamination } \\
\text { No }=3\end{array}$} & & \\
\hline & $\mathbf{N}$ & $\%$ & $\mathbf{N}$ & $\%$ & $\mathbf{N}$ & $\%$ & $\mathbf{N}$ & $\%$ & $\mathbf{N}$ & $\%$ & & \\
\hline $\begin{array}{l}\text { PSS } \\
\text { Mild } \\
\text { Moderate } \\
\text { Severe } \\
\text { Fatal }\end{array}$ & $\begin{array}{l}28 \\
35 \\
13 \\
10\end{array}$ & $\begin{array}{l}32.5 \\
39.3 \\
14.6 \\
11.2\end{array}$ & $\begin{array}{l}5 \\
8 \\
2 \\
0\end{array}$ & $\begin{array}{c}33.3 \\
53.4 \\
13.3 \\
0.0\end{array}$ & $\begin{array}{l}0 \\
2 \\
7 \\
2\end{array}$ & $\begin{array}{c}0.0 \\
18.2 \\
63.6 \\
18.2\end{array}$ & $\begin{array}{l}0 \\
1 \\
0 \\
0\end{array}$ & $\begin{array}{c}0.0 \\
100.0 \\
0.0 \\
0.0\end{array}$ & $\begin{array}{l}0 \\
2 \\
1 \\
0\end{array}$ & $\begin{array}{c}0.0 \\
66.7 \\
33.3 \\
0.0\end{array}$ & 21.03 & $\begin{array}{c}0.01 \\
S\end{array}$ \\
\hline $\begin{array}{l}\text { Outcome } \\
\text { Completely cured } \\
\text { Cured with } \\
\text { complication } \\
\text { Discharged against } \\
\text { medical advice } \\
\text { Death }\end{array}$ & $\begin{array}{l}10 \\
56 \\
11 \\
9 \\
9\end{array}$ & $\begin{array}{l}11.6 \\
65.1 \\
12.8 \\
10.5 \\
\end{array}$ & $\begin{array}{c}0 \\
11 \\
3 \\
1 \\
1\end{array}$ & $\begin{array}{c}0.0 \\
73.3 \\
20.0 \\
6.7 \\
\end{array}$ & $\begin{array}{l}2 \\
8\end{array}$ & $\begin{array}{l}18.2 \\
72.2 \\
0.0 \\
9.1 \\
\end{array}$ & $\begin{array}{l}0 \\
0\end{array}$ & $\begin{array}{r}0.0 \\
0.0 \\
0.0 \\
100.0 \\
\end{array}$ & $\begin{array}{l}0 \\
1 \\
2 \\
0 \\
0\end{array}$ & $\begin{array}{c}0.0 \\
33.3 \\
66.7 \\
0.0 \\
\end{array}$ & 19.7 & $\begin{array}{l}0.16 \\
\mathrm{NS}\end{array}$ \\
\hline $\begin{array}{l}\text { ICU admission } \\
\text { Yes } \\
\text { No }\end{array}$ & $\begin{array}{l}21 \\
65\end{array}$ & $\begin{array}{l}24.4 \\
75.6\end{array}$ & $\begin{array}{c}3 \\
12\end{array}$ & $\begin{array}{l}20 \\
80\end{array}$ & $\begin{array}{l}9 \\
2\end{array}$ & $\begin{array}{l}81.8 \\
18.1\end{array}$ & $\begin{array}{l}1 \\
0\end{array}$ & $\begin{array}{c}100 \\
0\end{array}$ & $\begin{array}{l}0 \\
3\end{array}$ & $\begin{array}{c}0 \\
100\end{array}$ & 27.1 & $\begin{array}{c}<0.001 \\
\text { HS }\end{array}$ \\
\hline
\end{tabular}


Table (10): Statistical analysis Fisher's exact test of the relation between severity grades of the studied cases according to PSS and poison type, need for ICU admission, duration of hospital stay and outcome $(\mathrm{NO}=116)$ :

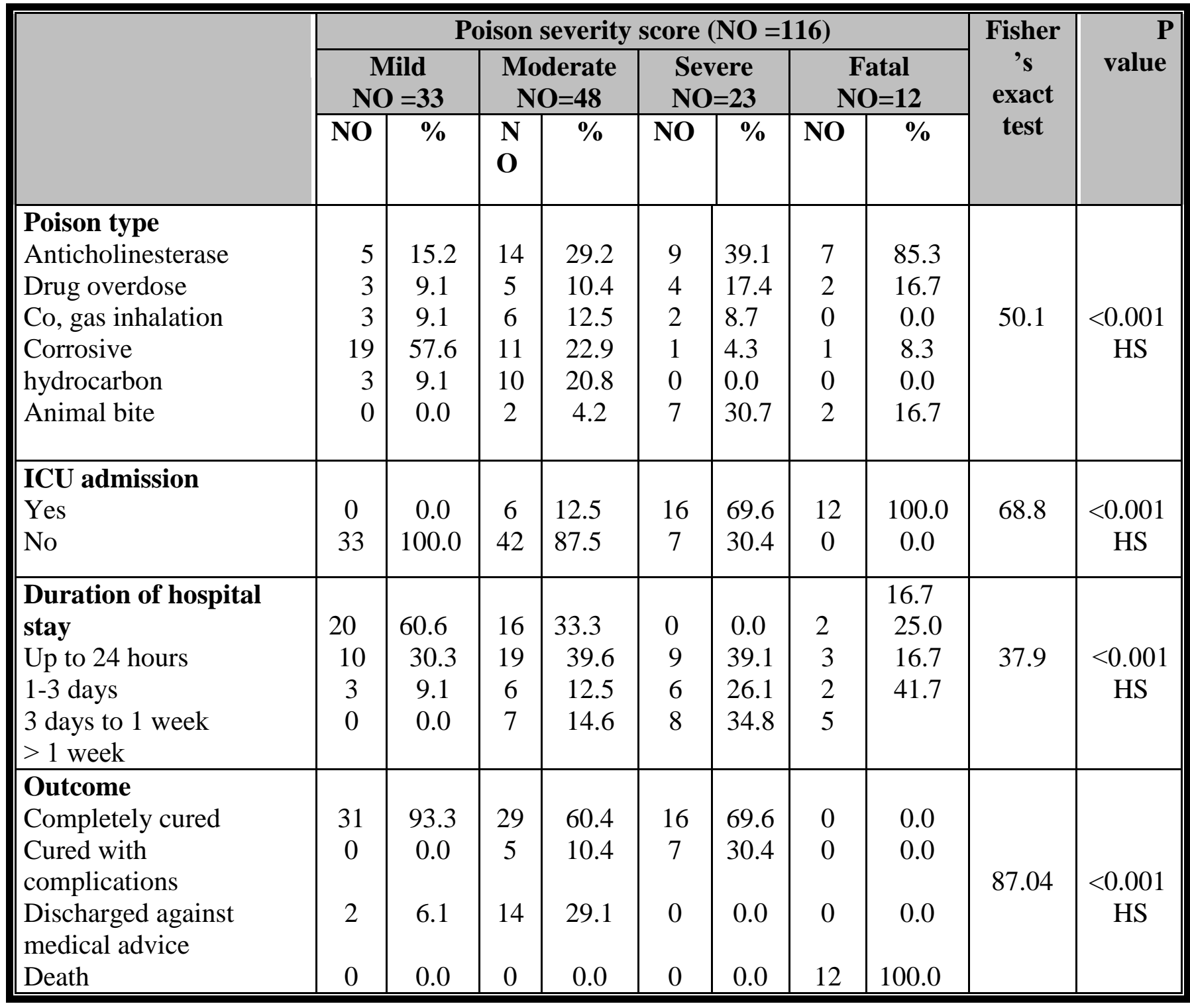

*NO= number of cases

${ }^{*} \mathrm{HS}=$ high significant

On studying the sex

This study is a prospective study carried on 116 acute poisoned cases suffered from respiratory system affection attended to MPDCC.

The most affected age group was children $<5$ years $(42.2 \%)$. More than half of patients were males $(54.3 \%)$ and the studied patients were mainly from rural areas $(60.3 \%)$ (Table 1$)$ differentiation in different age groups, there was statistically highly significant relation $(\mathrm{P}$ value $=0.001$ ) where females outnumbered males in age group $10-<20$ years $(34 \%$ versus $11.1 \%$ respectively) while males outnumbered females in age group 20-40 years and $>40$ years (30.2\% and $20.6 \%$ versus $9.4 \%$ and $7.5 \%$ respectively) (Table 2) 
Cholinesterase inhibitors and corrosive poisonings were the most common cause of respiratory system affection (30.2\% and $27.6 \%$ respectively) followed by drug overdose, hydrocarbons toxicity, animal bite toxicity and carbon monoxide \& other gases inhalation toxicity $(12.1 \%, 11.2 \%, 9.5 \%$ and $9.5 \%$ respectively).

Accidental exposure to poisoning was the most common mode of exposure $(79.3 \%)$ followed by suicidal poisoning $(20.7 \%)$ with no homicidal cases. The majority of cases was exposed to poison through oral route $(74.1 \%)$ followed by inhalation, biting, skin contamination and injection (12.9\%, $9.5 \%, 2.6 \%$ and $0.9 \%$ respectively).

Patients that came to MPDCC immediately within 3 hours after poison exposure represented $71.6 \%$ while $18.1 \%$ came within 3 to 6 hours and $10.3 \%$ came after 6 hours. $74.1 \%$ of cases were referred from other hospitals. Indoor was the major place of poison exposure (73.3\%). Most of poisoning exposure occurred in spring $(31.9 \%)$ and winter $(30.2 \%)$. (Table 3)

The most frequent presentations noticed in the studied cases were dyspnea $(59.5 \%)$, chest crepitation $(52.6 \%)$, cough $(37.1 \%)$, tachypnea $(25.9 \%)$ and hoarseness of voice (22.4\%). (Figure 2)

On studying the relation between types of respiratory presentation with age, there was statistically high significant relation between cough and different age groups where the most common cases presented with cough were in age group of $<5$ years old and there were statistically significant relations between dyspnea, chest pain, hoarseness of voice, wheezes and crepitation in different age groups. (Table 4)

On studying the relation between the presentations of the study cases with the causative poisons, it was observed that the relations between dyspnea, tachypnea, bradypnea, cough, dysphonia, wheezes and crepitation and the causative poisons were highly significant $(\mathrm{P}$ value $=<$ 0.001) while the relations were significant with chest pain and cyanosis. (Table 5)

Poison severity score was used to estimate the severity of the studied cases. The majority of cases were in moderate grade $(41.4 \%$ of cases), followed by mild, severe and fatal grades $(28.4 \%, 19.8 \%$ and $10.3 \%$ respectively) (Figure 3 ).

Regarding the investigations that were done to the studied cases, arterial blood gases were done to $70.7 \%$ of cases. Acidosis was noticed in $40.2 \%$ of them followed by mixed metabolic acidosis and respiratory alkalosis and respiratory alkalosis $(29.3 \%$ and $19.5 \%$ respectively). (Table 6)

Thin layer chromatography was done to $32.8 \%$ of cases, $84.2 \%$ of them had positive results. Pseudocholinesterase level was measured in $25.9 \%$ of cases and decreased levels were noticed in $96.7 \%$ of them. Rapid immunoassay detection kits were done to $13.8 \%$ of cases and $75 \%$ of them showed positive results (table 6)

Chest x-ray was done to $67.2 \%$ of cases, $53.8 \%$ of them showed 
abnormal findings as increased bronchovascular markings (19.2\%), pneumonic patches (29.5) and pneumothorax $(5.1 \%)$. Computed tomography (CT) of the chest was done to $15.5 \%$ of cases, $88.9 \%$ of them showed abnormal findings in the form of pulmonary consolidation $(72.2 \%)$ and pleural effusion (16.7\%) (Table 6) (Figures 4, 5 and 6)

Stroboscopy was done to $3.4 \%$ of cases, all of them showed abnormal findings as signs of laryngitis. (figure 7)

Table (7) shows statistically significant relation between the CT findings and the different age groups where most of abnormal findings were presented in $10-<20$ years and $>40$ years $(24 \%$ and $29.4 \%$ respectively). It was also noticed that statistically significant relation was found between age and causative poisons where corrosives and hydrocarbons were common in age of $<5$ years $(40.8 \%$ and $26.5 \%$ respectively) and cholinesterase inhibitors were common in age of $10-<20$ years.

On studying the relation between the ABG findings and age, the relation was significant where acidosis was found commonly in age of $<5$ years and 20-40 years (30.3\% for each), respiratory alkalosis was found commonly in the age of $<5$ years $(43.8 \%)$ but mixed acidosis and alkalosis was found in age of $10-<20$ years (37.5\%). (Table 8)

The relation between $\mathrm{ABG}$ findings and poison type was significant where acidosis was found commonly with cholinesterase inhibitors and drug overdose $(39.4 \%$ and $36.4 \%$ respectively) while respiratory alkalosis was found commonly with $\mathrm{CO}$ and other gas inhalation (31.3\%). (Table 8)

Regarding the cases' outcome, the majority of cases were completely cured $(65.5 \%)$, cure with complications was noticed in $10.3 \%$ (as chronic laryngitis (3.4\%), tracheostomy $(1.7 \%)$, ventilator associated pneumonia (2.6\%), dysphagia, esophageal stricture and surgical emphysema $(0.9 \%$ for each)) and mortality represented $10.3 \%$ of cases (the main cause of death was anticholinesterase poisoning (58.3\%)) (figure 8 )

This study reveals that the relation between the different routes of exposure and the severity grades of the studied cases was statistically significant where oral intake was the common in all severity grades while injection was the route in moderate grade cases only and skin contamination was the route in moderate and severe grade cases only (table 9)

The study also shows statistically high significant relation $(\mathrm{P}$ value $=<0.001)$ between the route of exposure to poison and the need for ICU admission where most of bitten cases $(81.8 \%)$ and the case who injected the poison were admitted in ICU while most of cases who ingested the poison didn't need admission in ICU (75.6\%). (Table 9)

Table (10) revealed statistically high significant relation between the severity grades of the studied cases and the poison type $(\mathrm{P}$ value $=$ $<0.001)$ where severe and fatal 
grades were commonly associated with anticholinesterase poisoning.

Our study showed statistically high significant relation between the severity grades of the cases and their need to ICU admission as all cases of fatal grade and $69.6 \%$ of severe grade were admitted to ICU $(\mathrm{P}$ value $=<0.001) .($ table 10$)$

This study showed statistically high significant relation between the severity grades of the studied cases according to PSS and the duration of their hospital stay $(\mathrm{P}$ value $=$ $<0.001$ ), as patients who stayed for up to 24 hours were mainly of mild and moderate grades $(60.6 \%$ and $33.3 \%$ respectively) while patients who stayed for more than one week were mainly of severe and fatal grades $(34.8 \%$ and $41.7 \%$ respectively).

The difference between the severity grades of cases and their outcome was statistically highly significant $(\mathrm{P}$ value $=<0.001)$, where most of mild $(93.3 \%)$ were cured completely while complicated cases were of moderate and severe grades $(10.4 \%$ and $30.4 \%$ respectively).

\section{DISCUSSION}

Respiratory system is often affected in acute poisoning as a lot of poisons can cause respiratory toxicity by several mechanisms. Several poisons may cause central or peripheral respiratory system affection in addition to acute poisoning complications as aspiration pneumonia and prolonged ventilation (Stolbach and Hoffman , 2011)

The incidence of respiratory system affection in acute poisoned cases through the period of the study was $4.2 \%$ as the total admitted cases were 2724 cases and the respiratory system affection was found only in 116 cases. Similarly to Abd-Elhaleem and Al Muqem, (2014) who observed respiratory symptoms in $3.5 \%$ of acute poisoned cases in their retrospective study in King Khaled Hospital in Al Majmaah Region, Saudi Arabia. Several studies reported different rates of respiratory symptoms and complications in acute poisoned cases as Kishore et al., (2008) who stated that respiratory system affection represented $10.56 \%$ of systems affection in their study of the pattern of poisoning cases in a teaching hospital in Western Nepal, Lund et al., (2012) who noticed that respiratory complications were the most recorded complications in his study of acute poisonings in adults $(\geq 16$ years) in Olso and Teklemariam et al., (2016) who found that $9.7 \%$ of patients of acute poisoning in Ethiopia were complaining from breathing difficulties.

Regarding age, most of patients were $<5$ years old. This may be due to children less than 5 years old are at high risk of exposure to acute poisoning because children are curious and try to explore the world with all their senses beside improper storage of medicines and household chemicals within reach of children (Dayasiri et al., 2017). Young children are in great risk of respiratory system affection in acute poisoning due to sensitive respiratory centers and high possibility of aspiration during 
vomiting. Also, the pediatric airway is narrow specifically the subglottic region so it can be easily obstructed by edema or secretions (Joshi and Ross, 2017).

The most common causative agent of respiratory affection in age below five years are corrosives and hydrocarbons, this comes in agreement with Madboly and Elgendy, 2014; Gangal and Haroon, 2015.

Regarding sex, males outnumbered females $(54.3 \%$ versus $45.7 \%$ respectively).

The high incidence of poisoning in males may be due to high exposure rates to poisoning in work places especially in fields due to insecticides handling during spraying trees and snake bites (Mate et al., 2017).

This study showed statistically significant relation between gender and different age groups. Females outnumbered males in age of 10$<20$ years while males outnumbered females in age of 20-40 years and $>40$ years. The increased incidence in males in this age group of $>20$ years may be due to they are the only earning members of their families so they are more vulnerable to stress as unemployment and marital problems in addition to occupational hazards to poisoning as insecticides and animal bites in fields, while increased incidence in females in age of 10-<20 years may be due to their vulnerability to stress during puberty, emotional status of young girls and domestic violence (Asawri et al., 2017)

The same results were observed by Association of Poison Control Centers' (AAPCC) and National
Poison Data System (NPDS) according to the annual report published in 2017 which stated that female predominance was found among poison exposure victims in age group of 13-19 years while male predominance was found in patients between 20 to 39 years old (Gummin et al., 2017)

Cases from rural areas outnumbered those from urban areas. This may be due to the rural nature of Menoufia governorate, where availability of pesticides and other toxic materials in home and fields beside the presence of snakes. Lack of awareness about children may attribute to high incidence of poisoning noticed in rural areas (ElGendy et al., 2008). This study comes in agreement with Hassan and Siam, (2014); Siva et al., (2015) and Ramesh et al., (2016).

This study revealed that, the most common poisons causing respiratory system affection were cholinesterase inhibitors $(30.2 \%)$ followed by corrosives $(27.6 \%)$.

Gangal and Haroon, (2015) noted that $1.34 \%$ of patients in their study suffered from respiratory distress and the most common toxic agents were insecticides followed by corrosives.

Cholinesterase inhibitors was reported to be the common cause of respiratory complications in many studies as Bhat et al., (2012); Ssemugabo et al., (2017); Nazima et al., (2018); Rao et al., (2018).

Chibishev et al., (2014) and Caganova et al., (2017) stated that corrosives poisoning are a common cause of poisoning induced pulmonary symptoms. 
Ingestion of the poison was the most common route of exposure $(74.1 \%)$. It may be because most of respiratory toxicants present in liquid form and easily to be ingested as cholinesterase inhibitors, corrosives, drugs and hydrocarbons (Coskun et al., 2015).

There was statistically significant relation between the different routes of exposure and the severity grades of the studied cases where oral intake was the common in all severity grades. This may be due to most of respiratory toxicants in this study are available in oral formulation and easily to be ingested.

There was statistically high significant relation $(\mathrm{P}$ value $=$ $<0.001$ ) between the route of exposure to poison and the need for ICU admission where most of bitten cases $(81.8 \%)$. This may be due to patients of animal bites specifically poisonous snake bites were more susceptible to respiratory muscle failure and they are in great need for mechanical ventilation in ICU

(Mehvish et al., 2016).

The same result was reported by Al-Barraq and Farahat (2011); Alazab et al., (2012); Mbarouk et al., (2017) and AAPCC, NPDS according to the annual report published in 2017 (Gummin et al., 2017).

Accidental poisoning was more frequent between the studied cases represented $79.3 \%$. Careless labeling and unsafe storage of poisonous substances may be causes of accidental poisoning, beside that lack of parental supervision may increase incidence of accidental poisoning specially in children (Oliveira and Suchara, 2014)

This is in agreement with Sahin et al., (2011); Hassan and Siam, (2014); Azab et al., (2016) and Seif et al., 2018. In contrary to this result, many studies stated that intentional poisoning is more than accidental as Boshehri et al., (2012); El-Masry and Tawfik, (2013) and Teklemariam et al., (2016)

It was found that the majority of the studied cases $(71.6 \%)$ sought medical advice within the first three hours of exposure. This may be because the population is aware that poisoned patients need an immediate medical care as acute poisoning is a medical emergency especially if associated with respiratory manifestations as dyspnea and cyanosis. The fear from these manifestations especially in children encourages the emergency transfer of the patients to hospital.

This is in agreement with Siddiqui et al., (2008); Ramesha et al., (2009); Manzar et al., (2010) and Saleem et al., (2015).

As regard referral data, $74.1 \%$ of the studied cases were referred from other health centers. This is may be due to that the MPDCC is known to be the only center treating poisoning cases in menoufia governorate so cases came rapidly to it.

Indoor exposure of poisoning represented $73.3 \%$ while $26.7 \%$ exposed to poison outdoor. The Annual Report of the American Association of Poison Control Centers' (AAPCC) National Poison 
Data System (NPDS) according to the published 2015 annual report found that $93.5 \%$ of cases exposure occurred at a residence (Mowry et al., 2015). Studies done by ElGendy et al., (2008); Hassan and Siam, (2014); Azab et al., (2016); Mbarouk et al., (2017) agree with these results.

As regard seasonal variation, most of cases were prevalent in spring $(31.9 \%)$ and winter $(30.2 \%)$. This may be due to spring is a season of agriculture in agricultural governorate as Menoufia with availability of pesticides and other toxic substances for agricultural purposes. (Ahmad et al., 2017). It may be also due to the maximum incidence of suicidal attacks is mainly in April and May as this period precedes examination in most schools and universities, which is characterized by high levels of stresses (Shreed et al., 2011). This is in agreement with Andýran and Sarýkayalar, (2004) and Wang et al., (2017). Moreover, increased prevalence in winter may be due to using of fired coal and stoves for warmth with high risk of carbon monoxide poisoning and hydrocarbon toxicity. This comes in agreement with Sahin et al., 2011).

As regard the clinical presentation of the studied cases on admission, dyspnea, chest crepitation (pulmonary odema), cough and tachypnea were the most common.

A retrospective study by Chibishev et al., 2014 of 415 patients at the University Clinic for Toxicology in Skopje, Republic of Macedonia for a period of five years, with this study as the most common noticed respiratory symptoms were difficult breathing, suffocation, cough, cyanosis and chest pain.

The same results also stated by Lifshitz et al., 2003; Madboly and Elgendy, 2014 and Anwar et al., 2014.

Regarding the cholinesterase inhibitors poisoning, this study noticed that pulmonary edema (chest crepitation) and dyspnea were the most frequent presentations $(91.4 \%$ and $74.3 \%$ respectively. Nazima et al., (2018) agreed with this result and stated that aspiration pneumonia and pulmonary edema were commonly found in organophosphorous patients in his study of management of organophosphorous poisoning patients in ICU in Srinagar, India during the period between2007 to 2009.

On studying the relation between types of presentations and the age of the studied cases, there was statistically high significant relation between cough and age where most of cases presented with cough were in age of $<5$ years.

Madboly and Elgendy, (2014) agreed with this result in their study at Benha university over one year 2013-2014 who stated that the most poisoned cases were below age of five and cough was the most frequent presentation. This may be due to children below five years try to put everything in their mouth with high risk of vomiting and aspiration which presented with respiratory irritation and cough.

According to poison severity score (PSS), the current study showed that most cases were of 
moderate grade $(41.4 \%)$ followed by mild grade and severe grade ( $28.4 \%$ and $19.8 \%$ respectively). Selection of poison severity score (PSS) for classification of the studied cases is as PSS is more comprehensive and appears to represent each system involved in a sequential progression. (Akdur et al., (2010); Abd El Salam et al., (2011)

Ebrahimi et al., (2018) in a Medical Research Center in Kermanshah, Iran stated that the majority of cases in their study were in grade 2 (moderate) which come in agreement with the current study.

On the contrary, Akdur et al., (2010) found that the majority of cases were in mild grade followed by severe grade and then moderate grades.

This study showed that, different abnormalities were noticed in arterial blood gases results. Liu et al., (2008) stated that, there was highly significant relation between the blood acidity (metabolic, respiratory and mixed) and severity and outcome of cases of organophosphorous poisoning.

This study noticed significant relations between $\mathrm{ABG}$ findings and the age of the studied cases and the poison type.

The present study revealed that respiratory acidosis occurred commonly with drug overdose. This comes in agreement with Rahimi et al., 2014 in his retrospective study in Loghman Hakim Hospital, Iran who stated that, pure respiratory acidosis was found in patients with acute tramadol poisoning and this may be due to respiratory center depression induced by tramadol.
Decreased serum cholinesterase levels were found mainly with cholinesterase inhibitors poisoning. An Indian study in 2008 reported that serum cholinesterase may be useful to assess the severity of the cases and prolonged duration of hospital stay (Rehiman et al., 2008)

Most of the studies have reported association of low serum acetylcholinesterase levels with the case severity (Bhattacharyya et al., 2011; Muley et al., 2014)

Abnormal chest X-ray findings as increased bronchovascular marking, pulmonary consolidation, pneumonic patches and pneumothorax were found mainly with corrosives, cholinesterase inhibitors and hydrocarbon poisonings and abnormal CT findings were found mainly with cholinesterase inhibitors poisoning.

The study of corrosive poisoning in adults by Chibishev et al., (2014) in Skopje, Republic of Macedonia agreed with the current study as it reported multiple abnormal $x$-ray findings as bronchopneumonia, pulmonary consolidation and pleural effusion.

In a case control study by Siddiqui et al., (2008) of sixty seven children with hydrocarbon ingestion in Aga Khan Hospital, Karachi, it was found that $57 \%$ of patients had radiological consolidation of lungs. This is in agreement with Lifshitz et al., (2003) and Sen et al., (2013).

Sun et al., (2015) reported that pneumonia was a very important prognostic factor of severity and outcome in 
cholinesterase inhibitor poisoning and abnormal radiological findings as pulmonary infiltrates were helpful in diagnosis of pneumonia during the hospital course.

As regarding the outcome of the studied cases, the majority of cases $(65.5 \%)$ were completely cured, while $10.3 \%$ of cases were cured with complication as dysphagia, tracheostomy and esophageal stricture.

Different studies report tracheostomy done among acute poisoning patients as Hiremanth et al., (2016) who report 21 patients $(56.7 \%)$ out of 37 patients with tracheostomy, whereas Kang et al., (2009) who report 17 patients $(25 \%)$ out of 68 patients with tracheostomy.

The mortality rate among the studied cases was $10.3 \%$, cholinesterase inhibitor poisoning was the most common cause of death $(53.3 \%)$. This coincides with Muley et al., (2014) who reported mortality of $10.56 \%$ in his study in India and Jamal et al., (2017) stated that the reported mortality rate was $9.7 \%$ in their study in Abassi Shaheed Hospital, Karachi.

Vaidya and Hulke, (2012) stated that overall mortality due to poisoning was $20 \%$. It was highest in insecticidal poisoning $(51.3 \%)$

This study showed statistically high significant relation between the severity grades of the cases and the poison type where cholinesterase inhibitors poisoning were the most common of causing severe and fatal toxicity. This result comes in agreement with Eddleston et al., (2006); Vaidya and Hulke,
(2012) and Ssemugabo et al., (2017).

This study showed that, there was statistically high significant relation between the severity grades of the cases and their need for ICU admission where most of severe $(69.6 \%)$ and all fatal grades cases admitted in ICU. This result were in agreement with Abd-El Salam et al., (2011) and Sam et al., (2009).

One of important indication of ICU admission is the requirement for mechanical ventilation (MV). Patients requiring mechanical ventilation due to respiratory depression usually need prolonged MV support. Excessive fatigue of respiratory muscles, central nervous system toxicity, hypopnea, aspiration of the gastric contents or even aspiration of active charcoal in the patients who do not have secure airways are major indications for MV (Acikalin et al., 2017)

The study demonstrated statistically high significant relation between the severity grades of the cases and the duration of hospital stay.

Ebrahimi et al., (2018)
stated that there was more correlation between the real duration of hospitalization and PSS (correlation coefficient $=0.23$ ) . Positive correlation indicated that increasing the poisoning severity in the sample led to increasing the criteria mean that cause increasing the hospitalization duration.

This study showed that, there was statistically high significant relation between the severity grades of the studied cases according to 
PSS and their outcome. This comes in agreement with a study done by Churi et al., (2012) and Hrabetz et al., (2013). Ebrahimi et al., (2018) who stated that, patient with the severity poisoning $\geq 2$ (moderate) might lead to death and the condition of the patients with the severity poisoning $<2$ (moderate) might lead to surviving.

\section{CONCLUSION}

The present study showed that respiratory system is commonly affected in cases of acute poisoning due to poison action or its complications. Respiratory system affection is a major cause of morbidity and mortality in acute poisoned cases. Cholinesterase inhibitors poisoning and corrosives are common respiratory toxicants. Children less than 5 years are more susceptible to acute poisoning induced respiratory manifestations.

\section{RECOMMENDATIONS}

Encouragement of early seeking medical advice in cases of acute poisoning especially if associated with respiratory system affection. Proper evaluation and management of poisoned cases should be done to decrease morbidity and mortality. All potentially poisonous chemicals, especially cholinesterase inhibitors and corrosives, should always be stored in secure containers and in a secure place away from children.

\section{REFERENCES}

Abd El Salam, H.; Fayed, A. M., \& Muneum, M. (2011): "Prediction of the outcome of patients with acute hydrocarbons poisoning using poison severity scoring system; a prospective study." J Am Sci, 7(4): 509-518.

Abd-Elhaleem, Z. A. E., \& Al Muqhem, B. A. (2014): "Pattern of acute poisoning in Al Majmaah region, Saudi Arabia." Am J Clin Exp Med, 2(4): 79-85.

Acikalin, A., Dişel, N. R., Matyar, S., Sebe, A., Kekec, Z., Gokel, Y., \& Karakoc, E. (2017): "Prognostic factors determining morbidity and mortality in organophosphate poisoning." Pakistan journal of medical sciences, 33(3): 534539.

Ahmad, I., Ahmad, S., Iqbal, W., Nazir, M., Ahmed, W., Kawoosa, K., \& Ali, S. W et al., (2017): " Spectrum, Complications and Outcome of Acute Pediatric Poisoning."Toxicology International, 24(1): 128-131.

Akdur, O., Durukan, P., Ozkan, S., Avsarogullari, L., Vardar, A., Kavalci, C., \& Ikizceli, I. (2010): " Poisoning severity score, Glasgow coma scale, corrected QT interval in acute organophosphate

poisoning." Human \& experimental toxicology, 29(5): 419-425.

Alazab, R. M., Elmougy, M. T., Fayad, R. A., Abdelsalam, H. F., \& Mohamed, A. S. (2012): "Risk factors of acute poisoning among children: A study at a poisoning unit of a university hospital in Egypt." South East Asia Journal of Public Health, 2(2): 41-47. 
Al-Barraq, A., and Farahat, F. (2011): "Pattern and determinants of poisoning in a teaching hospital in Riyadh, Saudi Arabia." Saudi Pharmaceutical Journal, 19(1): 57-63.

Andýran, N., and Sarýkayalar, F. (2004): "Pattern of acute poisonings in childhood in Ankara: what has changed in twenty years?." The Turkish journal of pediatrics, 46: 147152.

Anwar, S., Rahman, A. K. M. N., Houqe, S. A., Moshed, A. K. M. A., Yasmin, L., Saleh, A. S. M., \& Mohsin, M. (2014): "Clinical profile of kerosene poisoning in a tertiary level hospital in Bangladesh.", Bangladesh Journal of Child Health, 38(1): 11-14.

Asawari, R., Atmaram, P., Bhagwan, K., Priti, D., Kavya, S., \& Jabeen, G. A. (2017): "Toxicological Pattern of Poisoning in Urban Hospitals of Western India." Journal of Young Pharmacists, 9(3): 315320.

Azab, S. M., Hirshon, J. M., Hayes, B. D., El-Setouhy, M., Smith, G. S., Sakr, M. L., \& Klein-Schwartz, W et al., (2016): "Epidemiology of acute poisoning in children presenting to the poisoning treatment center at Ain Shams University in Cairo, Egypt, 20092013." Clinical toxicology, 54(1), 20-26.

Bhat, N. K., Dhar, M., Ahmad, S., \& Chandar, V. (2012): "Profile of poisoning in children and adolescents at a North Indian tertiary care centre." J Indian Acad Clin Med, 13(1): 37-42.

Bhattacharyya, K., Phaujdar, S., Sarkar, R., \& Mullick, O. S. (2011): "Serum creatine phosphokinase: A probable marker of severity in organophosphorus poisoning." Toxicology international, 18(2): 117-123.

Boshehri, B., Salimi, S., \& Ranjbar, S. (2012): "Mortality from acute poisoning in urmia: a three-year retrospective study." Iranian Red Crescent Medical Journal, 14(12): 838839.

Caganova, B., Foltanova, T., Puchon, E., Ondriasova, E., Plackova, S., Fazekas, T., \& Kuzelova, M. (2017): "Caustic ingestion in the elderly: influence of age on clinical outcome." Molecules, 22(10): 1726.

Chibishev, A. A., Simonovska, N., Bozinovska, C., Pereska, Z., Smokovski, I., \& Glasnovic, M. (2014): "Respiratory complications from acute corrosive poisonings in adults." Materia sociomedica, 26(2): 80-83.

Churi, S., Bhakta, K., \& Madhan, R. (2012): "Organophosphate poisoning: prediction of severity and outcome by Glasgow Coma Scale, poisoning severity score, Acute Physiology and Chronic Health Evaluation II score, and Simplified Acute Physiology Score II." Journal of emergency nursing, 38(5): 493-495. 
Coskun, R., Gundogan, K., Sezgin, G. C., Topaloglu, U. S., Hebbar, G., Guven, M., \& Sungur, M. (2015): "A retrospective review of intensive care management of organophosphate insecticide poisoning: Single center experience." Nigerian journal of clinical practice, 18(5): 644650.

Dayasiri M B, Jayamanne S F \& Jayasinghe C Y (2017): "Risk Factors for Acute Unintentional Poisoning among Children Aged 1-5 Years in the Rural Community of Sri Lanka." International journal of pediatrics, 1-9.

Ebrahimi, K., Raigani, A. A. V., Jalali, R., \& Rezaei, M. (2018): "Determining and comparing predictive and intensity value of severity scores-"Sequential organ failure assessment score," "Acute physiology and chronic health Evaluation 4," and "Poisoning severity score"in short-term clinical outcome of patients with poisoning in an ICU." Indian Journal of Critical Care Medicine, 22(6): 415.421.

El Masry, M. K., and Tawfik, H. M. (2013): "2011 Annual Report of the Poison Control Centre of Ain Shams University Hospital, Cairo, Egypt." Ain Shams J Forensic Med Clin Toxicol, 20(1): 10-17.

Eddleston, M., Mohamed, F., Davies, J. O., Eyer, P., Worek, F., Sheriff, M. R., \& Buckley, N. A. (2006): "Respiratory failure in acute organophosphorus pesticide self-poisoning." Journal of the Association Physicians, 99(8): 513-522.

El-Gendy, I. S., Hassan, N. A., \& Hegazy, R. M. (2008): "Frequency and Pattern of Poisoning Admission in Benha University Poison Center, Egypt: 2005-2006." East Mediterr Health J, Accepted on, 21st April

Gangal, R., and Haroon, A. (2015): "Profile of acute poisoning in paediatric age in district Moradabad: a hospital based study." Journal of Indian Academy of Forensic Medicine, 37(2): 155-159.

Gummin, D. D., Mowry, J. B., Spyker, D. A., Brooks, D. E., Fraser, M. O., \& Banner, W. (2017): "2016 Annual Report of the American Association of Poison Control Centers' National Poison Data System (NPDS): 34th Annual Report." Clinical toxicology, 55(10): 1072-1254.

Hassan, B. A., and Siam, M. G. (2014): "Patterns of acute poisoning in childhood in Zagazig, Egypt: an epidemiological study." International scholarly research notices, 1-5.

Hansen, N., Niederberger, A. S., Guzzella, L., \& Koumoutsakos, P. (2009): "A method for handling uncertainty in evolutionary optimization with an application to feedback control of combustion." IEEE Transactions on Evolutionary Computation, 13(1): 180-197.

Hrabetz, H., Thiermann, H., Felgenhauer, N., Zilker, T., 
Haller, B., Nährig, J., \& Eyer, F. (2013): "Organophosphate poisoning in the developed world-A single centre experience from here to the millennium." Chemico-

biological interactions, 206(3): 561-568.

Hiremath, P., Rangappa, P., Jacob, I., \& Rao, K. (2016): "Pseudocholinesterase as a predictor of mortality and morbidity in organophosphorus poisoning." Indian journal of critical care medicine: peerreviewed, official publication of Indian Society of Critical Care Medicine, 20(10): 601-604.

Jamal, Q., Rahman, A. S., Siddiqui, M. A., Riaz, M., \& Ansari, M. (2017): "Apache II Scoring as an Index of Severity in Organophosphorus

Poisoning." J

Clin

Toxicol, 7(354): 2161-0495.

Jayakrishnan, B., Al Asmi, A., Ahmed Al Qassabi, R. N., \& Mohammed, I. (2012): "Acute drug overdose: clinical profile, etiologic spectrum and determinants of duration of intensive medical treatment." Oman medical journal, 27(6): 501-504.

Joshi, P., and Ross, M. P.(2017): Intensive care of pediatric poisoning cases in: Brent $\mathrm{J}$ et al, $2^{\text {nd }}$ ed. Critical care toxicology. Springer, USA; 205-222.

Kang, E. J., Seok, S. J., Lee, K. H., Gil, H. W., Yang, J. O., Lee, E. Y., \& Hong, S. Y. (2009): "Factors for determining survival in acute organophosphate

poisoning." The Korean journal of internal medicine, 24(4): 362-367.

Khodabandeh, F,. and Agin, $\mathbf{K}$. (2016): "Assessment of aspiration induced lung injuries among acute drug poisoning patients, Longhman Hakim hospital poisoning center." International Journal of medical toxicology and forensic medicine, 6(4): 209-216.

Kishore, P. V., Paudel, S. P. R., Mishra, D., Ojha, P., Alam, K., \& Mishra, P. (2008): "Pattern of poisoning cases in a teaching hospital in Western Nepal." Journal of Institute of Medicine, 30(1): 26-34.

Lifshitz, M., Sofer, S., \& Gorodischer, R. (2003): "Hydrocarbon poisoning in children: a 5-year retrospective study." Wilderness $\&$ environmental medicine, 14(2): 78-82.

Little M. (2015): Approach to the poisoned patient, toxicology emergencies in: Cameron $\mathrm{P}$ et al., $4^{\text {th }}$ ed. Text book of adult emergency medicine. Elsevier 2015: 951-957.

Liu, J. H., Chou, C. Y., Liu, Y. L., Liao, P. Y., Lin, P. W., Lin, H. H., \& Yang, Y. F. (2008): "Acid-base interpretation can be the predictor of outcome among patients with acute organophosphate poisoning before hospitalization." The American journal of emergency medicine; 26(1): 24-30.

Lund, C., Drottning, P., Stiksrud, B., Vahabi, J., Lyngra, M., Ekeberg, I., \& Hovda, K. E et al., (2012): "A one-year observational study of all 
hospitalized acute poisonings in Oslo: complications, treatment and sequelae." Scandinavian journal of trauma, resuscitation and emergency medicine, 20(1): 49.

Madboly, A. G., and Elgendy, F. S. (2014): "Epidemiology, Clinical Characteristics, and Management of Acute Hydrocarbons Poisoning at Benha Poisoning Control Unit: A One-Year Prospective Clinical Study.", Ain Shams J. Foren. Med. Clin. Toxicol, 23: 30-42.

Malangu, $\mathbf{N}$ and Ogunbanjo, G.A (2009): "A profile of acute poisoning at selected hospitals in South Africa, South African." Journal of Epidemiology Infection; 24: 4-16.

Manzar, N., Saad, S. M. A., Manzar, B., \& Fatima, S. S. (2010): "The study of etiological and demographic characteristics of acute household accidental poisoning in children-a consecutive case series study from Pakistan." BMC pediatrics, 10(1): 28.

Mate VH, Dhande PP, Gonarkar SB, Pandit VA.(2017): "A Prospective Observational Study on Pattern, Severity and Outcome of Different Poisoning Cases in a Tertiary Care Hospital, India." J Basic Clin Pharma, 8(3):154- 157.

Mbarouk, G. S., Sawe, H. R., Mfinanga, J. A., Stein, J., Levin, S., Mwafongo, V., \& Olson, K. R et al., (2017): "Patients with acute poisoning presenting to an urban emergency department of a tertiary hospital in Tanzania." BMC research notes, 10(1): 482.

Mehvish, H., Mustafa, M., Fairrul, K., EL.IIIzam., Firdaus, H., Nornazirah, A.,(2016): "Neurotoxicity related to snakebite: treatment, and prevention.", Journal of Medical and Dental Science Research, 3(10): 20-25

Mowry J, Spyker D, Brooks et al., (2015): "Annual Report of the American Association of Poison Control Centers' (AAPCC) National Poison Data System (NPDS) 2014: 32 $2^{\text {nd }}$ Annual Report." Clinical Toxicology; 53(10): 962-1146.

Muley A, Shah C, Lakhani J, Bapna M, Mehta J.(2014): "To identify morbidity and mortality predictors in acute organophosphate poisoning." Indian J Crit Care Med ;18(5): 297-300.

Nazima, S. N., Bashir, Y., Nabi, S., \& Bashir, N. (2018): "Intensive care management of organophosphorus poisoning patients: an experience from tertiary care centre." International Journal of Advances in Medicine, 5(2): 257-264.

Oliveira F F S and Suchara E A (2014): "Epidemiological profile of exogenous poisoning in children and adolescents from a municipality in the state of Mato Grosso." Revista Paulista de Pediatria; 32(4): 299-305.

Olson, K. R. (2012): Comprehensive evaluation and 
treatment in: Olson K R et al., $6^{\text {th }}$ eds. Olson's Poisoning \& Drug Overdose. McGraw Hill; 47.

Persson, H.; Sjöberg, G.; Haines J, Pronczuk, de Garbin J. (1998): "Poisoning Severity Score: Grading of acute poisoning." J Toxicology Clinical Toxicology, 36: 205213.

Rahimi, H. R., Soltaninejad, K., \& Shadnia, S. (2014): "Acute tramadol poisoning and its clinical and laboratory findings." Journal of research in medical sciences: the official journal of Isfahan University of Medical Sciences, 19(9): 855859.

Ramesha, K. N.; Rao, K. B.\& Kumar, G. S. (2009): 'Pattern and outcome of acute poisoning cases in a tertiary care hospital in Karnataka, India." Indian J Crit Care Med, 13; 152-155.

Ramesh, V., Chavan, V. R., Arshad, M., \& Raghunandan, n, K. B., \& Dinleyici, E. C. (2011): "Acute poisoning in children; data of a pediatric emergency unit." Iranian journal of pediatrics, 21(4): 479-484.

Saleem, A., Ejaz, M. S., Arif, F., Hanifa, A., \& Habib, M. I. (2015): "Factors leading to acute accidental poisoning in children." Quarterly Medical Channel; 21(4): 5-9.

Sam, K. G., Kondabolu, K., Pati, D., Kamath, A., Kumar, G. P., \& Rao, P. G. (2009): "Poisoning severity score, APACHE II and GCS: effective clinical indices for estimating severity and predicting outcome
M. (2016): Faizuddin. "A study on Pattern of Acute Poisoning in an Emergency Department of a Tertiary Care Hospital." Asian Journal of Pharmaceutical and Clinical Research, 9(3): 361363.

Rao, K. N., Babu, P. S., \& Acharya, A. (2018): "A prospective study of pattern of acute poisoning and treatment outcome in paediatric age group in tertiary care hospital of Konaseema region of Andhra Pradesh, India." International Journal of Contemporary Pediatrics, 5(2): 576-579.

Rehiman, S., Lohani, S. P., \& Bhattarai, M. C. (2008): "Correlation of serum cholinesterase level, clinical score at presentation and severity of organophosphorous poisoning." J Nepal Med Assoc, 47(170): 47-52.

Sahin,

$$
\text { S., }
$$

Carma

of acute organophosphorus and carbamate poisoning." Journal of forensic and legal medicine, 16(5): 239-247.

Seif, E., Gomaa, R., \& Eisa, M. (2016): "A retrospective study of acute poisoning in children under 5 years old admitted to Alexandria Poison Center in Egypt." World journal of preventive medicine, 4(2): 3239.

Sen, V., Kelekci, S., Selimoglu Sen, H., Yolbas, I., Gunes, A., Abakay, O., \& Fuat Gurkan, M. (2013): "An evaluation of cases of pneumonia that occurred secondary to 
hydrocarbon exposure in children." Eur Rev Med Pharmacol Sci, 17(Suppl 1): 912.

Shreed, S., Tawfik, N., Mohammed, N., \& Elmahdi, M. (2011): "Toxic agents used for parasuicide in Damietta Governorate, Egypt." Middle East Current Psychiatry, 18(1): 11-17.

Siddiqui, E., Razzak, J., Naz, F., \& Khan, S. J. (2008): "Factors associated with hydrocarbon ingestion in children." Journal of the Pakistan Medical Association, 58(11): 608-612.

Siva, s.; Reddy, T. M.; Ahammad, S. F. \& Durga, T. S. (2015): "Pattern of acute poisoning, therapeutic approach and outcome in South Indian teaching hospitah, Andhra Pradesh." International journal of Universal Pharmacy and Bio Science, 4(6): 67-78.

Ssemugabo, C., Halage, A. A., Neebye, R. M., Nabankema, V., Kasule, M. M., Ssekimpi, D., \& Jørs, E. (2017): "Prevalence, circumstances, and management of acute pesticide poisoning in hospitals in Kampala City, Uganda." Environmental health insights, 11: 1-8.

Stolbach, A. and Hoffman, R. S. (2011): Respiratory principles in: Goldfrank L R et al., $9^{\text {th }}$ ed. Goldfrank's toxicologic emergencies. McGraw-Hill Education; 303-313.

Sun, I. O., Yoon, H. J., \& Lee, K. Y. (2015): "Prognostic factors in cholinesterase inhibitor poisoning." Medical science monitor: international medical journal of experimental and clinical research, 21: 29002904.

Teklemariam, E., Tesema, S., \& Jemal, A. (2016): "Pattern of acute poisoning in Jimma University Specialized Hospital, South West Ethiopia." World journal of emergency medicine, 7(4): 290-293.

Vaidya, Y. P., and Hulke, S. M. (2012): "Study of trends of poisoning in the cases reported to government hospital, Yavatmal." Chronicles of Young Scientists, 3(1): 63-67.

Wang, L., Tang, Y., Hu, R., Xue, Z., \& Zheng, J. (2017): "Epidemiologic characteristics of poisoning among hospitalized children in Shanxi, a north Chinese city during 2008-2013." Int J Clin Exp Med, 10(5): 8183-8191. 


\section{الملخص العربي}

تأثر الجهاز التنفسى فى حالات التسمم الحاد التى تصل اللى مركز علاج التسمم والإدمان

بستثفيات جامعة المنوفية " دراسة مستقبلية "1

أ.د/سامى عبدالهادى حماد1، أ.د/عزة وجيه زناتى²، أ.م.د/علا عبدالهادى سويلم³، د/فاطمة شعبان

قنديل4، ،ط/ريهام صبحى أبوعياد 5

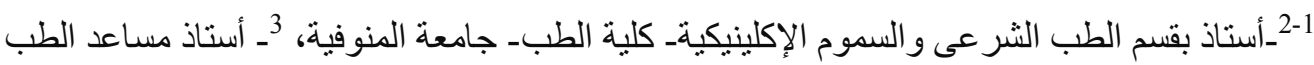

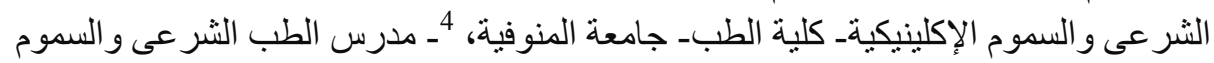

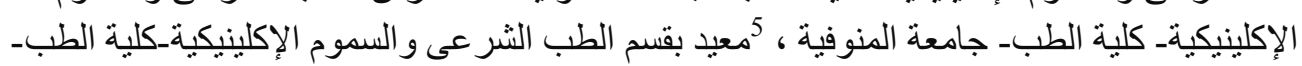

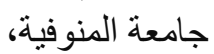

المقدمة: يعد التسمم الحاد احدالاسباب الثهيرة للاخول فى قسم الطوارىه وقد يحتاج إلى الدخول

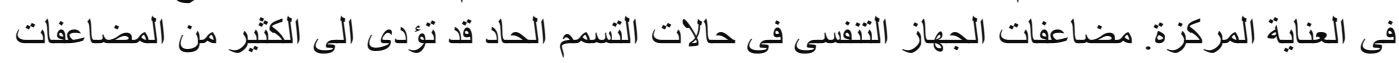
المرضية وكذلك الوفاة.

الهدف من الاراسة: دراسة تأثر الجهاز التنفسى فى حالات التسمح الحاد من حيث الأنماط

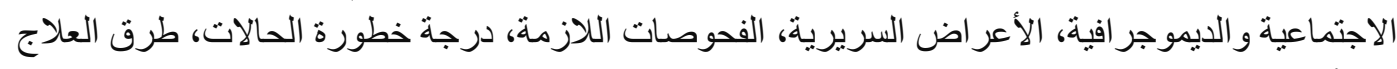

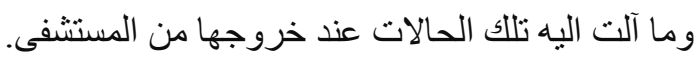

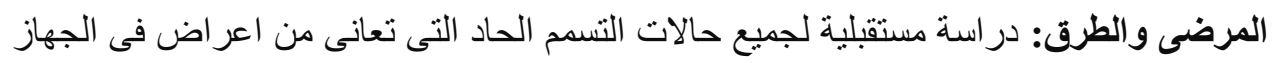

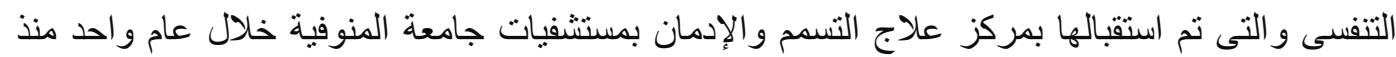

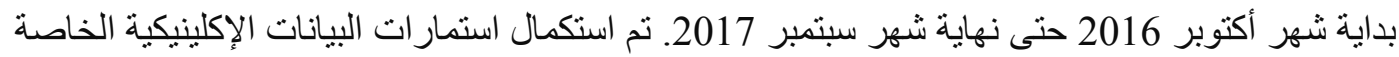

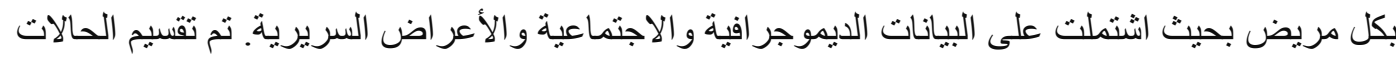
على حسب درجة خطورنهاز

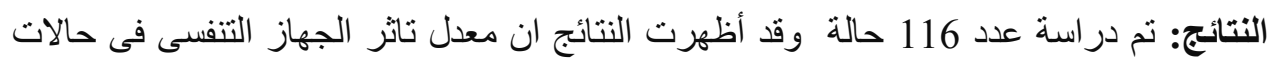

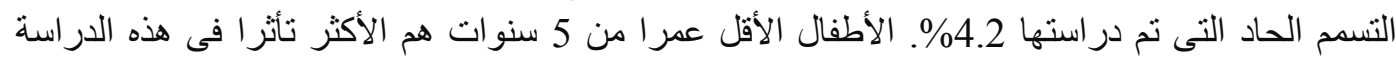

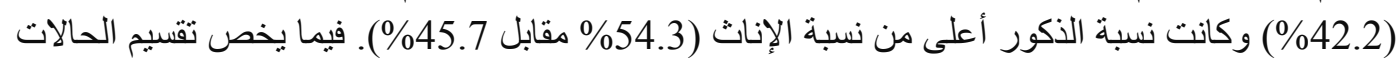

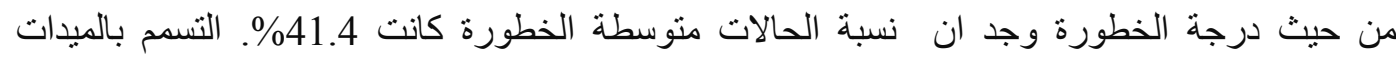

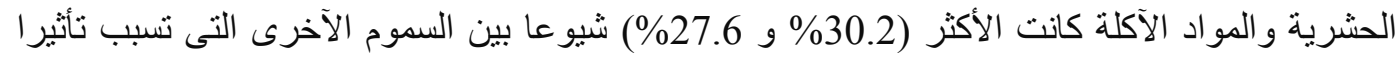

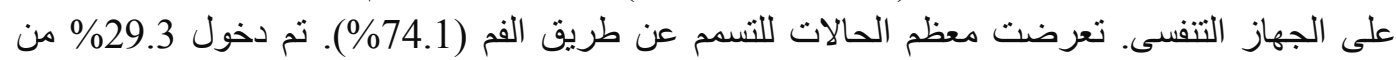
الحالات فى وحدة العناية المركزة وكانت نسبة حدوث الوفاة كانت 10.3 \%

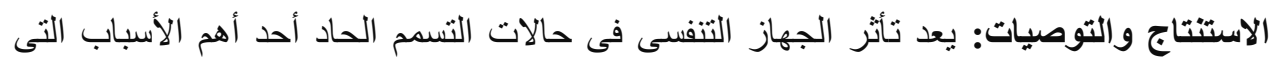

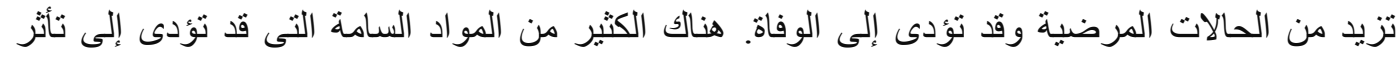

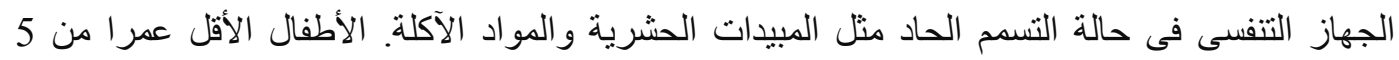

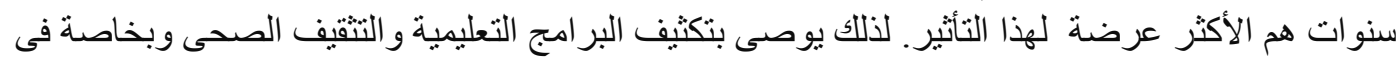
المناطق الريفية وكذلك حظر تداول المبيدات الحشرية والمنتجات المنزلية الآخرى التى تسبب التسمم التية وحفظها بعيدا عن متناول الأطفال. 